\title{
On Vibration and Noise Dissipation in Ship and FPSO Structures with Smart Systems
}

\author{
Vincent O. S. Olunloyo ${ }^{1}$ and Charles A. Osheku ${ }^{2}$ \\ ${ }^{1}$ Department of Systems Engineering, Faculty of Engineering, University of Lagos, Akoka-Yaba, Lagos 23401, Nigeria \\ ${ }^{2}$ Centre for Space Transport and Propulsion, National Space Research and Development Agency, \\ Federal Ministry of Science and Technology FCT, Abuja, PMB 437, Nigeria
}

Correspondence should be addressed to Charles A. Osheku, charlesosheku2002@yahoo.com

Received 10 January 2012; Accepted 12 March 2012

Academic Editors: C. F. Gao, R. Ohayon, and G.-J. Wang

Copyright ( 2012 V. O. S. Olunloyo and C. A. Osheku. This is an open access article distributed under the Creative Commons Attribution License, which permits unrestricted use, distribution, and reproduction in any medium, provided the original work is properly cited.

\begin{abstract}
Ships and floating structure production systems are widely deployed for deep and ultradeep waters operation. Active vibration reduction and noise control in such structures can significantly improve their hydrodynamic performance and stability during navigation, exploration, and exploitation activities. One way to minimise or reduce the transmission of vibration in these moving offshore structures is to exploit the mechanism of interfacial slip in press fit joints or layered structural laminates in their internal hull configurations to dissipate vibration energy. In this paper, slip damping with heterogeneous sandwich composite viscoelastic beam-plate smart systems as a model for dissipation of vibration and active noise control mechanism in ship and floating structures is investigated. For this problem, a boundary value partial differential equation is formulated for the case of linear and nonlinear hydrodynamic wave loadings. In particular, the effect of pressure distribution variation at the interface of the layered smart system on the energy dissipation, logarithmic damping decrement, and spatial transfer function is analyzed and presented for design application and selection of appropriate stabilizers.
\end{abstract}

\section{Introduction}

Ships and floating structures are widely employed in the course of deep and ultradeep waters navigation as well as oil and gas engineering operations, because of their high adaptability, relatively low construction cost, and good stability. With the renewed interest in off-shore oil and gas exploration, huge resources are being invested in the development of the structures.

These structures (mostly ship shaped) are either purposely built or merely converted from existing tankers with good hull structural scantling design. One of the factors that influence the design of these structures is the environment in which they have to operate. Nevertheless, FPSOs have unique features and characteristics. One new innovation in the design of ships and floating structures employs the application of sandwich plate system (SPS) technology with builtin vibration and insulation mechanism, as illustrated in Figure 1 . The structures are usually sited at specific locations and are subjected to dynamic loading that is quite different from those arising from unrestricted service conditions, Moan et al. [1].

In particular, such structures and systems are constantly under the influence of hydrodynamic forces that prevail in such locations. Within the context of the design of these structures and systems, most of the analyses currently employ probabilistic, statistical, and empirical models along with validating experiments and field observations. This makes the analysis and design of ships, floating production systems (FPS), and FPSOs a challenging process. One problem such designs have to address is adequate damping of vibration and active noise generation or transmission.

Vibration damping and active noise control in engineering structures and systems have attracted the attention of mathematical physicist and engineers for many years, (Lazan [2]; Nakra [3]; Sun and Lu [4]; Garibaldi and Onah [5]; Meador and Mead [6]; Vydra and Shogren [7]). With the application of surface damping and laminates in ships, the 


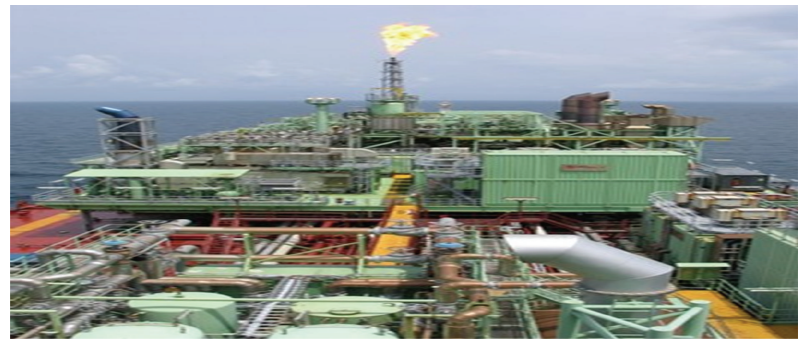

FIGURe 1: Sandwich Plate System (SPS) technology. Source: Intelligent Engineering (IE), UK/Canada.

FPS and the FPSOs are part of recent outcome of advancement in manufacturing processes that are cost-effective and suitable for high volume production. Contrary to the conventional laminating press procedure, it is now possible to manufacture multilayered damped laminates consisting of two metal skins with a viscoelastic core via continuous process in coil form using existing equipment and technology.

Several methods, classified into active, semiactive, and passive control methods, have, over the years, been effectively adapted to control noise and reduce vibration in various dynamic systems. Certain active elements have been employed in the past, such as speakers, actuators, and microprocessors to produce an "out-of-phase" signal that can electronically cancel the disturbance. For fluid-borne noise, passive control methods that have been deployed include the use of elements such as absorbers, barriers, mufflers, and silencers. Whilst for structurally borne vibration and noise, changing of the systems modal parameters can significantly alter the resonance frequencies, provided the excitation frequencies remain constant. Nonetheless, the most effective means for handling this problem is the vibration damping or isolation through the use of damping materials. In fact, semi-active methods use active control methods such as electrorheological (ER), magnetorheological (MR) fluids and active constrained layer damping (ACLD) where the traditional constraining layer is replaced with a smart structure to enhance the damping properties of passive elements as discussed by Rao [8].

The application of piezoelectric materials in the control of vibration and noise in flexible structures has also been a subject of investigation for several decades. Piezoelectric ceramics provide a cheap, reliable, and nonintrusive means of actuation and sensing in flexible structures. Such a structure commonly described in the literature as a smart or intelligent/adaptive system is simply constructed by sandwiching piezoelectric actuators and/or sensors with flexible or elastic structures; Moheimani et al. [9] and Bailey and Hubbard [10] provide excellent reviews. The above referenced materials strain when exposed to a voltage or alternatively produce voltage when strained as pointed out by Alberts et al. [11]. For detailed descriptions of the unique electromechanical behaviour of these materials refer to Tzou [12].

Historically, the mechanism of damping as a means of controlling the undesirable effects of vibration has been treated over the years either in the context of aerodynamic/hydrodynamic structures or within the machine tool industry. In fact, there are several ways of effecting such damping, including the introduction of constrained, unconstrained, and even viscoelastic layers. One such technique is layered construction made possible by externally applied pressure that holds the members together at the interface. Under such circumstances, the profile of the interface pressure assumes a significant role, especially in the presence of slip, to dissipate the vibration energy. Another way of getting rid of unwanted vibration is through material damping as characterized by the strain energy of the structural members.

Within the context of the cantilever beam, one of the earliest works on slip damping is attributed to Goodman and Klumpp [13]. The nature of the interface pressure profile across the beam layer is a separate and important issue that has also received some attention over the years. There are several ways of simulating such interfacial pressure including mechanisms such as bonded (welded) connections, or the use of bolted connections, and even bonded-bolted connections placed at appropriate locations along the laminate interface.

With the introduction of composite materials and the possible beneficial effects these materials can have on slip damping, several authors have revisited the problem of layered or jointed structures subjected to uniform pressure distribution. In this regard, Nanda [14] studied the effect of structural members under controlled dynamic slip while Nanda and Behera [15] examined the problem of slip damping of jointed structures with connection bolts as found in machine structures. One of the difficulties encountered in earlier analysis of this problem is the assumption of the uniform pressure profile at the interface of the layers, as experiments and earlier analysis had clearly shown that this was rarely the case. The effect of nonuniform interface pressure distribution on the mechanism of slip damping for layered elastic beams was recently examined by Damisa et al. $[16,18]$, and Olunloyo et al. [17]. In particular, whereas the investigation by Damisa et al. [16] was limited to the case of the linear pressure profile, the analysis in Olunloyo et al. [17] included other forms of interfacial pressure distributions such as polynomial or hyperbolic representations. In addition, the investigation in Damisa et al. [18] has also been extended to account for the effects of frequency of excitation and viscoelastic coefficients on the damping properties of layered viscoelastic beam plate in Olunloyo et al. [19]. The results obtained in Olunloyo et al. [17] demonstrated that the effects of nonlinearities in interfacial pressure distributions as compared with the linear profile were largely incremental in nature, and no fundamental differences were found. In a related development, the effects of smart layers and material properties on the damping properties of sandwich heterogeneous elastic beams with varying thicknesses were reported in Olunloyo et al. [20]. The results from these earlier studies provide adequate justification for the linear pressure profile selected for the present investigation.

This paper is organized as follows. Section 1 introduces the problem under investigation with appropriate notations. In the next section, the essential fluid-structure interaction 
mechanics is discussed. In Section 3, these relationships are incorporated into an analytical model for the computation of the response of the smart system under harmonic excitation. In Section 4, the energy dissipation is computed while Section 5 gives the procedural method for the computation of logarithmic damping decrement. Section 6 is concerned with analysis of spatial transfer functions for active vibration and noise control design. Following in Section 7 are the simulated results showing the modulating role of the piezoelectric parameter with respect to design analysis and application. Finally, in Section 8, the paper ends with the summary and conclusion.

\section{Essential Fluid-Structure Interaction Mechanics}

For an isentropic (ocean state) inviscid fluid flow problem, flow in the domain of fluid-structure interface for the case of linear hydrodynamic wave loading is governed by the differential equations

$$
\begin{gathered}
\nabla^{2} \Phi=0, \\
\frac{P_{h}}{\rho_{w}}+\frac{\partial \Phi}{\partial t}+g z+\frac{1}{2}|\nabla \Phi|^{2}=0 .
\end{gathered}
$$

In view of the elliptical hull geometry as illustrated in Figures 2(a) and 2(b) under consideration, the well-known transformation to take us from Cartesian $\left(x_{0}, y_{0}, z_{0}\right)$ to elliptical cylindrical coordinates $\left(\xi, \eta_{0}, z_{0}\right)$ holds, namely,

$$
x_{0}=c \cosh \xi \cos \eta_{0}, \quad y_{0}=c \sinh \xi \sin \eta_{0}, \quad z_{0}=z_{0},
$$

where $\xi \geq 0,0 \leq \eta_{0}<2 \pi,-\infty<z_{0}<\infty$.

Thereafter, the first part of (1a) takes the form

$$
\frac{1}{c^{2}\left(\sinh ^{2} \xi+\sin ^{2} \eta_{0}\right)}\left(\frac{\partial^{2} \Phi}{\partial \xi^{2}}+\frac{\partial^{2} \Phi}{\partial \eta_{0}^{2}}\right)+\frac{\partial^{2} \Phi}{\partial z_{0}^{2}}=0 .
$$

The decomposition of $\Phi$ into $\Phi=\phi\left(\xi, \eta_{0}\right) f\left(z_{0}, t\right)$ via variable separation leads to a set of two equations namely,

$$
\frac{1}{c^{2}\left(\cosh 2 \xi-\cos 2 \eta_{0}\right)}\left(\frac{\partial^{2} \phi\left(\xi, \eta_{0}\right)}{\partial \xi^{2}}+\frac{\partial^{2} \phi\left(\xi, \eta_{0}\right)}{\partial \eta_{0}{ }^{2}}\right)+k^{2} \phi\left(\xi, \eta_{0}\right)=0,
$$

$$
\frac{\partial^{2} f\left(z_{0}, t\right)}{\partial z^{2}}-k^{2} f\left(z_{0}, t\right)=0
$$

where $k^{2}$ is a constant (wave number) to be determined from the fluid kinematics boundary conditions. The solution of (4) within the context of fluid structure interaction boundary conditions is reported in Olunloyo et al. [21], while, $\phi\left(\xi, \eta_{0}\right)$, which can be further decomposed as $\phi\left(\xi, \eta_{0}\right)=F(\xi) G$, leads to the well-known Mathieu and the modified Mathieu differential equations, namely,

$$
\begin{gathered}
\frac{d^{2} G\left(\eta_{0}\right)}{d \eta_{0}^{2}}+\left(\bar{a}-2 \bar{q} \cos 2 \eta_{0}\right) G\left(\eta_{0}\right)=0, \\
\frac{d^{2} F(\xi)}{d \xi^{2}}-(\bar{a}-2 \bar{q} \cosh 2 \xi) F(\xi)=0,
\end{gathered}
$$

here the separation constant $\bar{a}$ is any characteristic eigenvalue while $\bar{q}=k^{2} c^{2} / 4$.

The solution to (6) and (7) is strongly influenced by the numerical value of $\bar{q}$ and for deep and ultradeep water problems $k$ is significantly small. Under such circumstances, the regime of linear and weakly nonlinear wave propagation can be satisfactorily assumed as the first-order approximation without loss of generality. In the meantime, (6) and (7) become amenable to parameter perturbation method. Consequently, $F(\xi), G(\eta)$, and $\bar{a}$ can all be expanded in perturbation series in terms of the parameter $\bar{q}$.

While noting the above, it is sufficient to state that the hydrodynamic wave loading force around a vibrating and translating ship or floating structure during exploration and exploitation or naval surveillance must satisfy the expression

$$
\vec{F}_{h}=\iint_{S} P_{h} \hat{e}_{z} d S
$$

where

$$
P_{h}=-\rho_{w}\left(\phi\left(\xi, \eta_{0}\right) \frac{\partial f\left(z_{0}, t\right)}{\partial t}+g z+\frac{1}{2} U^{2}\right)
$$

and $\hat{e}_{z}$ is the outward normal unit vector for the element $d S$ of the surface of the ship or floating structure.

Here, $U$ is the translational velocity of the approaching ocean water for the case of a vibrating localised ship or floating structure or relative translational velocity for a moving ship or floating structure. For such a problem, a closedform expression for $f\left(z_{0}, t\right)$ in the Laplace domain has been reported by Olunloyo et al. [21-23] and can be written as

$$
\tilde{f}\left(z_{0}, s\right)=-s \frac{\widetilde{W}\left(x_{0}, y_{0}, s\right)}{k \sinh \left(k h_{D}\right)} \cosh \left(k z_{0}\right),
$$

where $\widetilde{W}\left(x_{0}, y_{0}, s\right)$ and $h_{D}$ represent the dynamic interaction response and the depth of immersion of the ship or floating structure, respectively.

For the case of nonlinear hydrodynamic wave loading force, the appropriate modifications in (9) to the $o\left(\varepsilon_{s}{ }^{2}\right)$ yield

$$
\begin{aligned}
P_{h}=-\rho_{w} & \left(\left(\phi_{0}\left(\xi, \eta_{0}\right)+\varepsilon_{s} \phi_{1}\left(\xi, \eta_{0}\right)\right)\right. \\
& \left.\times \frac{\partial\left(f_{0}\left(z_{0}, t\right)+\varepsilon_{s} f_{1}\left(z_{0}, t\right)\right)}{\partial t}+g z+\frac{1}{2}\left(U_{0}^{2}+\varepsilon_{s} U_{0}^{2}\right)\right)
\end{aligned}
$$

while

$$
\begin{aligned}
& \tilde{f}_{0}\left(z_{0}, s\right)=-s \frac{\widetilde{W}_{0}\left(x_{0}, y_{0}, s\right)}{k_{0} \sinh \left(k_{0} h_{D}\right)} \cosh \left(k_{0} z_{0}\right), \\
& \tilde{f}_{1}\left(z_{0}, s\right)=-s \frac{\widetilde{W}_{1}\left(x_{0}, y_{0}, s\right)}{k_{1} \sinh \left(k_{1} h_{D}\right)} \cosh \left(k_{1} z_{0}\right),
\end{aligned}
$$

as reported by Olunloyo et al. [23]. 
Thus, the amplitude of the loading force $F_{0}$ as illustrated in Figure 3(a) must satisfy the expression

$$
F_{0} \geq \max \cdot\left\|\vec{F}_{h}\right\| .
$$

\section{Problem Definition}

As illustrated in Figure 3(a), the proposed sandwich composite smart structure consists of four layers of unequal thickness of the same length and width, respectively. In this case, the upper piezoelectric layer (actuator) and the lower piezoelectric layer (sensor) are assumed to be perfectly bonded to the surfaces of two dissimilar layers of elastic beam-plate structures. Furthermore, piezoelectric layers are also assumed to be of much smaller thickness than those of the respective laminates to which they are bonded. That is, $h_{a} \ll h_{1} ; h_{s} \ll h_{2}$. On the other hand, the proposed internal hull configuration is contrived as an assembly of cascade of composite smart structure layers as illustrated in Figure 3(b).

The problem here is to examine analytically the effect of the nature of load, ocean water frequency variation, piezoelectric variables, and the pressure gradient on

(i) the dynamic response of the clamped sandwich composite cantilever elastic beamplate,

(ii) the profile of interfacial slip,

(iii) the slip energy dissipation under dynamic conditions,

(iv) logarithmic damping decrement associated with mechanism of slip damping in such layered structures,

(v) spatial transfer function for vibration and active noise control design.

After excitation, the postslip geometries for both the upper and lower-layers are shown in Figures 3(c) and 3(d). Now for the contrived boundary value problem, the transverse displacement $W$, same for each layer, satisfies the following PDE:

$$
\begin{gathered}
\left(\frac{c_{1}+c_{2}}{4}\right) \frac{\partial^{5} W}{\partial t \partial x^{4}}+\frac{\partial^{4} W}{\partial x^{4}} \\
+\frac{\left(\beta_{1} \mathcal{A}+\beta_{2} \mathcal{B}+\beta_{3}+\beta_{4}\right)}{4} \frac{\partial^{2} W}{\partial t^{2}} \\
=\frac{\left(\alpha_{1} \mathcal{A}+\alpha_{2} \mathcal{B}\right)}{4} \frac{\partial P(x, 0)}{\partial x}+\frac{C_{a}}{4} \frac{\partial^{2} V_{a}}{\partial x^{2}},
\end{gathered}
$$

where $\mathcal{A}$ denotes $1-v_{1}^{2}$ and $\mathscr{B}$ denotes $1-v_{2}^{2}$, and the following parameters have been defined viz:

$$
\begin{gathered}
\alpha_{1}=\frac{6 \mu}{E_{\mathrm{eq}_{1}} h_{\mathrm{eq}_{1}}^{2}}, \quad \beta_{1}=\frac{\rho_{1} b h_{1}}{E_{1} I_{1}}, \\
\beta_{3}=\frac{\rho_{a} b h_{a}}{E_{a} I_{a 1}}, \quad h_{\mathrm{eq}_{1}}=\left(h_{1}+h_{a}\right), \\
\alpha_{2}=\frac{6 \mu}{E_{\mathrm{eq}_{2}} h_{\mathrm{eq}_{2}}^{2}}, \quad \beta_{2}=\frac{\rho_{2} b h_{2}}{E_{2} I_{2}}, \\
\beta_{4}=\frac{\rho_{s} b h_{s}}{E_{s} I_{s}}, \quad h_{\mathrm{eq}_{2}}=\left(h_{2}+h_{s}\right), \\
C_{a}=\frac{1}{2} E_{a} d_{31} b\left(h_{a}+h_{s}\right),
\end{gathered}
$$

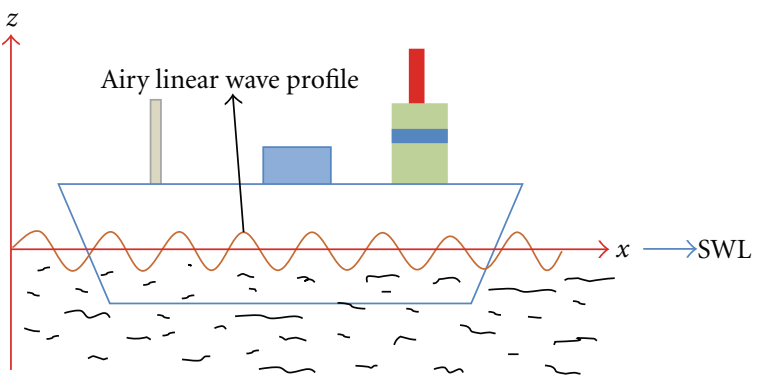

(a)

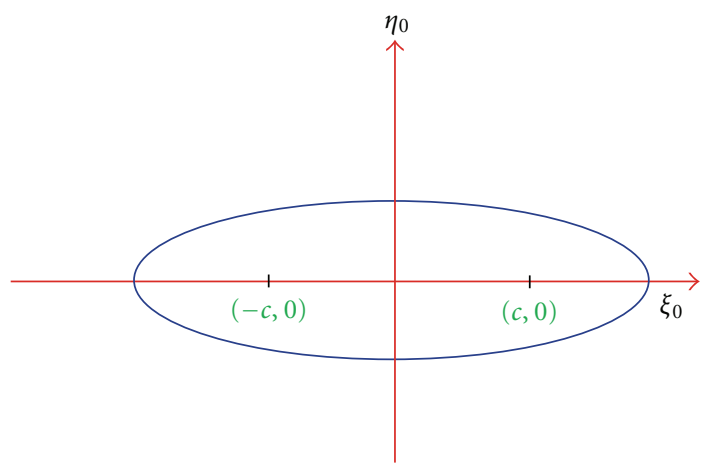

(b)

FIgURE 2: (a) A mathematical model of ship/FPSO in ocean environment. (b) An elliptical model representation of a ship/FPSO bottom hull configuration.

following Alberts et al. [11], here, $d_{31}$ is the electric charge constant of the film in $(\mathrm{m} / \mathrm{v})$.

We next introduce the Laplace transform, namely,

$$
\overline{(\bullet)}=\int_{0}^{\infty}(\bullet) e^{-s t} d t, \quad(\bullet)=\frac{1}{2 \pi i} \int_{\eta-i \infty}^{\eta+i \infty} \overline{(\bullet)} e^{s t} d s
$$

on (13) to obtain

$$
\begin{aligned}
& \left(1+s\left(\frac{c_{1}+c_{2}}{4}\right)\right) \frac{d^{4} \widetilde{W}(x, s)}{d x^{4}} \\
& +\frac{\left(\beta \mathcal{A}+\beta_{2} \mathcal{B}+\beta_{3}+\beta_{4}\right)}{4} \\
& \quad \times\left(s^{2} \widetilde{W}(x, s)-s W(0)-\dot{W}(0)\right) \\
& =\frac{\left(\alpha_{1} \mathcal{A}+\alpha_{2} \mathcal{B}\right)}{4 s} \frac{\partial P(x, 0)}{\partial x}+\frac{C_{a}}{4} \frac{d^{2} \widetilde{V}(x, s)_{a}}{d x^{2}} .
\end{aligned}
$$

By limiting the analysis to the case of linear pressure variation along the laminate interface, namely,

$$
P(x, 0)=P_{0}\left(1+\frac{\varepsilon}{L} x\right),
$$




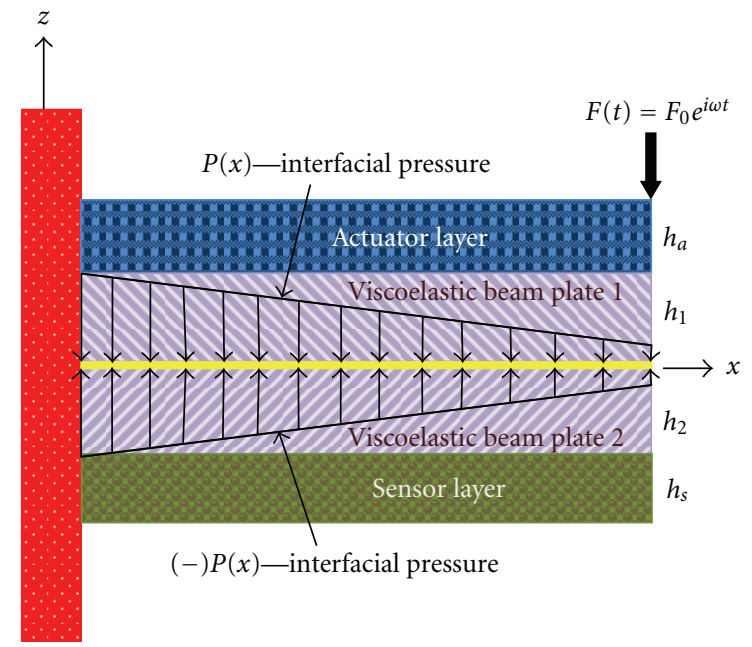

(a)

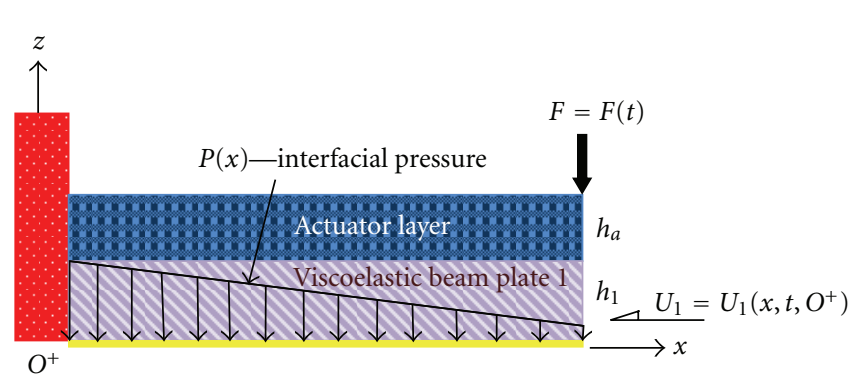

(c)

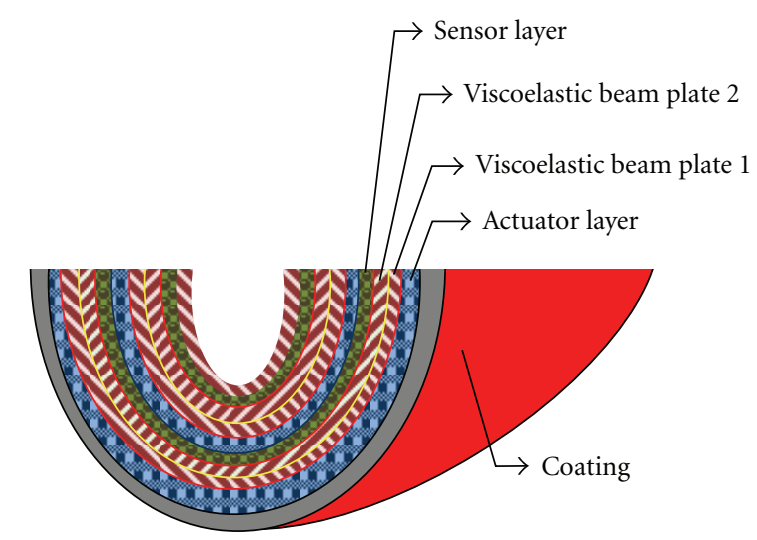

(b)

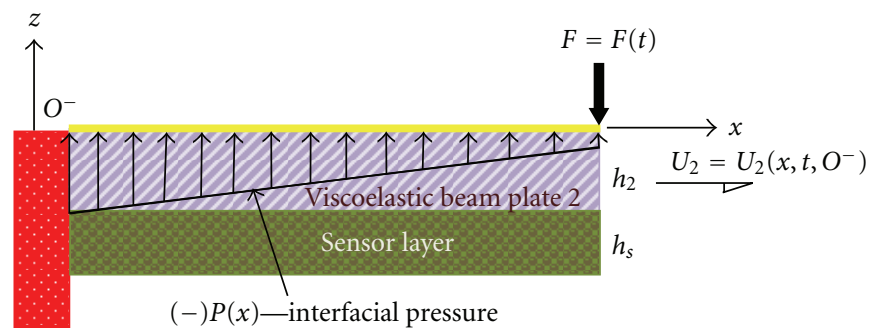

(d)

Figure 3: (a) Preslip geometry for the composite sandwich structure under dynamic load. (b) Layering cross-section of composite structure. (c) Upper layer postslip geometry under dynamic load. (d) Lower layer postslip geometry under dynamic load.

Equation (16) simplifies to the form

$$
\begin{aligned}
(1+ & \left.s\left(\frac{c_{1}+c_{2}}{4}\right)\right) \frac{d^{4} \widetilde{W}(x, s)}{d x^{4}} \\
+ & \frac{\left(\beta_{1} \mathcal{A}+\beta_{2} \mathscr{B}+\beta_{3}+\beta_{4}\right)}{4} \\
& \times\left(s^{2} \widetilde{W}(x, s)-s W(0)-\dot{W}(0)\right) \\
& =\frac{\left(\alpha_{1} \mathcal{A}+\alpha_{2} \mathscr{B}\right) \varepsilon P_{0}}{4 s L}+\frac{C_{a}}{4} \frac{d^{2} \widetilde{V}_{a}(x, s)_{a}}{d x^{2}} .
\end{aligned}
$$

In (18), the spatial distribution of the actuating layer is considered to be uniform with respect to $x$. Under this assumption, the term $d^{2} \tilde{V}_{a}(x, s)_{a} / d x^{2}$ following Alberts et al. [11] becomes

$$
\frac{d^{2} \widetilde{V}_{a}(x, s)_{a}}{d x^{2}}=\frac{\left(\delta^{\prime}(x-0)-\delta^{\prime}(x-L)\right) V_{a}}{s},
$$

where $\delta^{\prime}(\cdot)$ is the first derivative of the Dirac delta function. Introducing the Fourier finite sine transform

$$
\overline{[\bullet}]=\int_{0}^{L}[\bullet] \sin \frac{n \pi x}{L} d x, \quad[\bullet]=\frac{2}{L} \sum_{n=1}^{\infty} \overline{[\bullet]} \sin \frac{n \pi x}{L}
$$

in conjunction with the following boundary conditions in the Laplace transform plane namely,

$$
\widetilde{W}(0, s)=\frac{d}{d x} \widetilde{W}(0, s)=\frac{d^{2}}{d x^{2}} \widetilde{W}(L, s)=0
$$

gives the Fourier sine transform of (18) as

$$
\begin{aligned}
& \frac{n^{4} \pi^{4}}{L^{4}}\left(1+s\left(\frac{c_{1}+c_{2}}{4}\right)\right) \widetilde{W}^{F}\left(\lambda_{n}, s\right) \\
& +\frac{\left(\beta_{1} \mathcal{A}+\beta_{2} \mathscr{B}+\beta_{3}+\beta_{4}\right)}{4} s^{2} \widetilde{W}^{F}\left(\lambda_{n}, s\right) \\
& =\frac{\left(\alpha_{1} \mathcal{A}+\alpha_{2} \mathscr{B}\right)}{4 s} \frac{P_{0} \varepsilon}{n \pi}\left(1+(-1)^{n+1}\right) \\
& +\frac{n^{3} \pi^{3}}{L^{3}}(-1)^{n+1} \widetilde{W}(L, s)-\frac{n \pi}{L} \widetilde{W}_{x x}(0, s) \\
& \quad+\frac{C_{a} V_{a}}{4 s} n \pi\left(1+(-1)^{n+1}\right) .
\end{aligned}
$$


Following the procedure outlined in Olunloyo et al. [21], the bending moment is computed as:

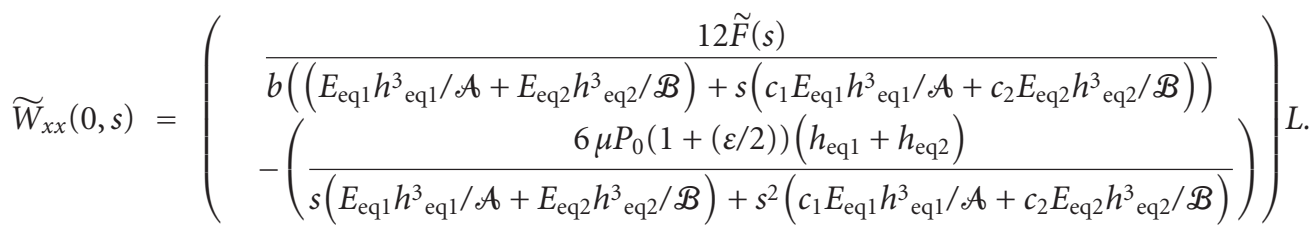

This result clearly indicates that the value for expression (25) cannot be fully determined until the forcing function $F(t)$ is specified. Next, we limit the analysis to the case of harmonic loading function. For this case, the forcing function is $F(t)=$ $F_{0} e^{i \omega t}$ with the driving transform as

$$
\widetilde{F}(s)=\frac{F_{0}}{s-i \omega},
$$

where $F_{0}$ represents the amplitude of the loading force and, $\omega$ is the maximum associated excitation frequency for the fluidstructure systems.

Recalling the only remaining unutilized boundary condition in (21), namely, $(d / d x) \widetilde{W}(0, s)=0$ the Fourier inversion of (22) leads to the expression

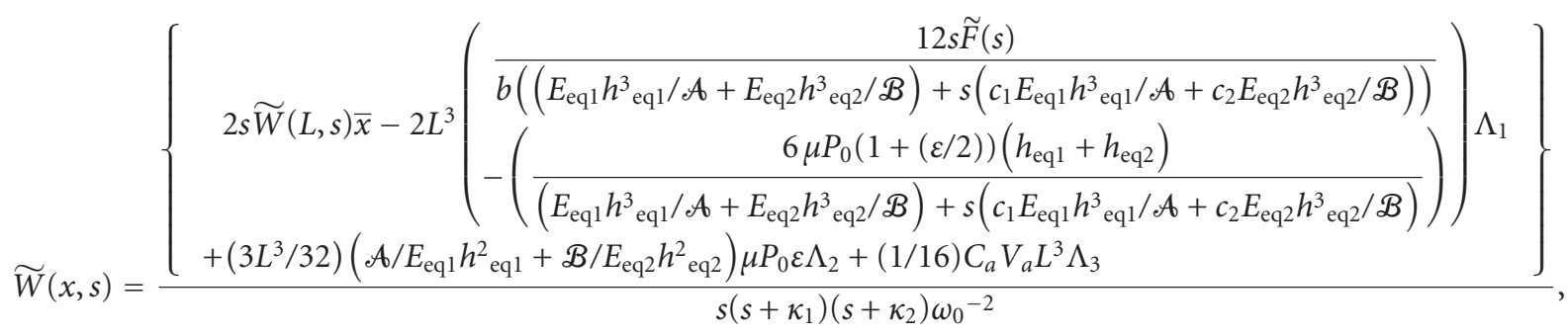

where

$$
\begin{gathered}
\kappa_{1}=\frac{\left(c_{1}+c_{2}\right) \omega_{0}^{2}}{8}+i \sqrt{\omega_{0}^{2}-\left(\frac{c_{1}+c_{2}}{8}\right)^{2} \omega_{0}^{4},} \\
\kappa_{2}=\frac{\left(c_{1}+c_{2}\right) \omega_{0}^{2}}{8}-i \sqrt{\omega_{0}^{2}-\left(\frac{c_{1}+c_{2}}{8}\right)^{2} \omega_{0}^{4},} \\
\omega_{1}^{2}=\omega_{0}^{2}\left(1-\frac{\left(c_{1}+c_{2}\right)^{2}}{32} \omega_{0}^{2}\right), \quad \omega_{0}^{2}=\frac{n^{4} \pi^{4}}{\bar{\beta} L^{4}} .
\end{gathered}
$$

Here,

$$
\begin{gathered}
\bar{\beta}=\frac{\left(\beta_{1} \mathcal{A}+\beta_{2} \mathcal{B}+\beta_{3}+\beta_{4}\right)}{4}, \\
\Lambda_{1}=\left(\frac{\bar{x}}{6}-\frac{\bar{x}^{2}}{4}+\frac{\bar{x}^{3}}{12}\right), \quad \Lambda_{2}=\left(\frac{\bar{x}}{45}-\frac{2 \bar{x}^{3}}{9}+\frac{\bar{x}^{4}}{3}-\frac{2 \bar{x}^{5}}{15}\right), \\
\Lambda_{3}=\left(\frac{\bar{x}}{3}-\frac{\bar{x}^{2}}{2}+\frac{\bar{x}^{3}}{6}\right) .
\end{gathered}
$$

Appropriate substitution for $\widetilde{W}(L, s)$ into (25) and rearrangement gives

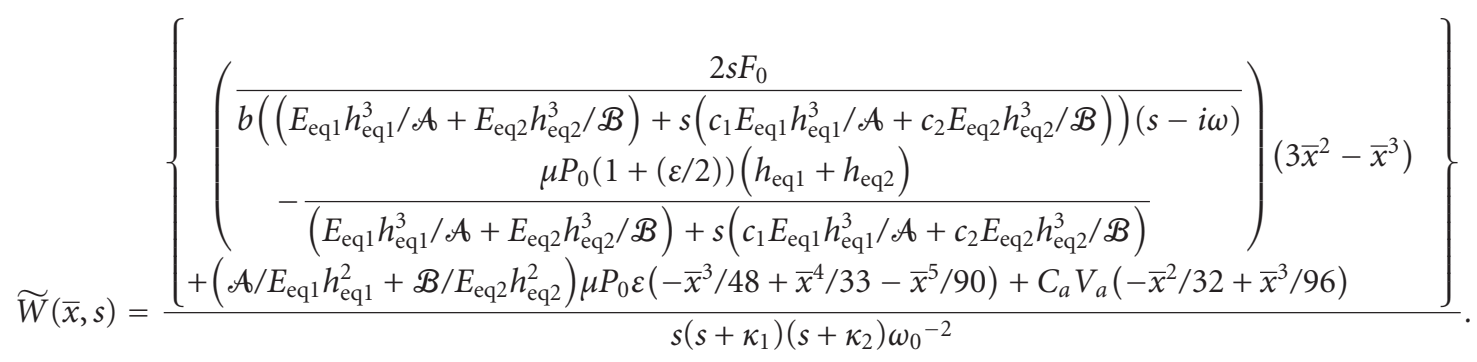

By employing the Laplace inversion we obtain the dynamic response as 
$\bar{W}(\bar{x}, \tau)$

$$
=\left(\begin{array}{c}
\left(2 F_{2}(\tau)-\mu \bar{P}_{0} \mathcal{C} F_{1}(\tau)\right)\left(3 \bar{x}^{2}-\bar{x}^{3}\right) \\
+\mu \bar{P}_{0}\left[-\frac{3 \mathcal{C}}{2} F_{1}(\tau) x^{2}+\left(\left(\frac{\mathcal{C}}{2}\right) F_{1}(\tau)-\left(\frac{\mathcal{A}+\mathcal{B} \gamma_{\mathrm{eq}}^{-1} \Psi_{\mathrm{eq}}^{-2}}{48}\right) F_{3}(\tau)\right) x^{3}\right] \varepsilon \\
+F_{3}(\tau)\left[\left(\mathcal{A}+\mathcal{B} \gamma_{\mathrm{eq}}^{-1} \Psi_{\mathrm{eq}}^{-2}\right)\left(\frac{x^{4}}{33}-\frac{x^{5}}{90}\right)\right]+\bar{C}_{a} F_{1}(\tau)\left(\frac{-\bar{x}^{2}}{32}+\frac{\bar{x}^{3}}{96}\right)
\end{array}\right),
$$

where

$$
t=\frac{2 \pi \tau}{\omega_{0}}, \quad \eta=\frac{\omega}{\omega_{0}}
$$

while

$$
\begin{aligned}
F_{1}(\tau)= & {\left[\left(\frac{\mathcal{A B}}{\mathcal{B}+\gamma_{\mathrm{eq}} \Psi_{\mathrm{eq}}^{3} \mathcal{A}}\right)+\frac{e^{-2 \pi \bar{\kappa}_{1} \tau}}{\bar{\kappa}_{1} \bar{\kappa}_{2} \bar{\kappa}_{12}}-\frac{e^{-2 \pi \bar{\kappa}_{1} \tau}}{\bar{\kappa}_{4} \bar{\kappa}_{5} \bar{\kappa}_{12}}\right.} \\
& \left.-\frac{\omega^{-1} e^{-2 \pi \eta \bar{\kappa}_{1} \tau}}{\bar{\kappa}_{13}}\right], \\
F_{2}(\tau)= & {\left[\frac{e^{-2 \pi \bar{\kappa}_{1} \tau}}{\bar{\kappa}_{2} \bar{\kappa}_{3}}+\frac{e^{-2 \pi \bar{\kappa}_{4} \tau}}{\bar{\kappa}_{3} \bar{\kappa}_{5}}+\frac{e^{i 2 \pi \eta \tau}}{\bar{\kappa}_{6} \bar{\kappa}_{7}}+\frac{\omega^{-1} \bar{\kappa}_{11} e^{i 2 \pi \eta \tau}}{\bar{\kappa}_{9} \bar{\kappa}_{10}}\right], } \\
F_{3}(\tau)= & {\left[1+\frac{e^{-2 \pi \bar{\kappa}_{1} \tau}}{\bar{\kappa}_{1} \bar{\kappa}_{12}}+\frac{e^{-2 \pi \bar{\kappa}_{4} \tau}}{\bar{\kappa}_{4} \bar{\kappa}_{12}}\right] . }
\end{aligned}
$$

Here,

$$
\begin{aligned}
& \bar{\kappa}_{1}=\left(\frac{\bar{\mu}_{1}+\bar{\mu}_{2}}{8 \eta}\right)-i \sqrt{1-\left(\frac{\bar{\mu}_{1}+\bar{\mu}_{2}}{8 \eta}\right)^{2}}, \\
& \bar{\kappa}_{2}=\left[\frac{\left(\mathscr{B}+\gamma_{\mathrm{eq}} \Psi_{\mathrm{eq}}^{3} \mathcal{A}\right)+\bar{\kappa}_{1}\left(\left(\bar{\mu}_{1} / \eta\right) \mathscr{B}+\left(\bar{\mu}_{2} / \eta\right) \mathcal{A} \gamma_{\mathrm{eq}} \Psi_{\mathrm{eq}}^{3}\right)}{(\mathcal{A B B})}\right] \text {, } \\
& \bar{\kappa}_{3}=\left[\frac{1}{32}\left(\frac{\bar{\mu}_{1}+\bar{\mu}_{2}}{\eta}\right)^{2}\right. \\
& \left.+\left(2 \eta+i\left(\frac{\bar{\mu}_{1}+\bar{\mu}_{2}}{4 \eta}\right)\right) \sqrt{\left(1-\left(\frac{\bar{\mu}_{1}+\bar{\mu}_{2}}{8 \eta}\right)^{2}\right)}-2\right], \\
& \bar{\kappa}_{4}=\left(\frac{\bar{\mu}_{1}+\bar{\mu}_{2}}{8 \eta}\right)+i \sqrt{1-\left(\frac{\bar{\mu}_{1}+\bar{\mu}_{2}}{8 \eta}\right)^{2}}, \\
& \bar{\kappa}_{5}=\left[\frac{\left(\mathscr{B}+\gamma_{\mathrm{eq}} \Psi_{\mathrm{eq}}^{3} \mathcal{A}\right)+\bar{\kappa}_{4}\left(\left(\bar{\mu}_{1} / \eta\right) \mathscr{B}+\left(\bar{\mu}_{2} / \eta\right) \mathscr{A} \gamma_{\mathrm{eq}} \Psi_{\mathrm{eq}}^{3}\right)}{(\mathcal{A B B})}\right] \text {, } \\
& \bar{\kappa}_{6}=\left[\left(1-\eta^{2}\right)-i\left(\frac{\bar{\mu}_{1}+\bar{\mu}_{2}}{4 \eta}\right)\right] \text {, } \\
& \bar{\kappa}_{7}=\left[\frac{\left(\mathcal{B}+\gamma_{\mathrm{eq}} \Psi_{\mathrm{eq}}^{3} \mathcal{A}\right)+i\left(\bar{\mu}_{1} \mathcal{B}+\bar{\mu}_{2} \mathcal{A} \gamma_{\mathrm{eq}} \Psi_{\mathrm{eq}}^{3}\right)}{(\mathcal{A B B})}\right] \text {, }
\end{aligned}
$$

$$
\begin{aligned}
& \bar{\kappa}_{8}=\left[\frac{\left(\mathcal{B}+\gamma_{\mathrm{eq}} \Psi_{\mathrm{eq}}^{3} \mathcal{A}\right)}{\left(\bar{\mu}_{1} \mathscr{B}+\bar{\mu}_{2} \mathcal{A} \gamma_{\mathrm{eq}} \Psi_{\mathrm{eq}}^{3}\right)}\right], \\
& \bar{\kappa}_{9}=\left[\bar{\kappa}_{8}^{2} \eta^{2}+\bar{\kappa}_{8}\left(\frac{\bar{\mu}_{1}+\bar{\mu}_{2}}{4 \eta}\right)+1\right], \\
& \bar{\kappa}_{10}=-\left\lfloor\left(\mathcal{B}+\gamma_{\mathrm{eq}} \Psi_{\mathrm{eq}}^{3} \mathcal{A}\right)+i\left(\bar{\mu}_{1} \mathcal{B}+\bar{\mu}_{2} \mathcal{A} \gamma_{\mathrm{eq}} \Psi_{\mathrm{eq}}^{3}\right)\right\rfloor, \\
& \bar{\kappa}_{11}=-\left\lfloor\left(\bar{\mu}_{1} \mathcal{B}+\bar{\mu}_{2} \mathcal{A} \gamma_{\mathrm{eq}} \Psi_{\mathrm{eq}}^{3}\right)\right\rfloor, \\
& \bar{\kappa}_{12}=2 i\left[\left(1-\left(\frac{\bar{\mu}_{1}+\bar{\mu}_{2}}{8 \eta}\right)^{2}\right),\right. \\
& \bar{\kappa}_{13}=-\left[\bar{\kappa}_{8}+\left(\frac{\bar{\mu}_{1}+\bar{\mu}_{2}}{4}\right)^{2}+\bar{\kappa}_{8}^{3}\right],
\end{aligned}
$$

where the following nondimensionalized parameters have also been introduced viz:

$$
\bar{W}(\bar{x}, \tau)=\frac{W(\bar{x}, \tau) E_{\mathrm{eq} 1} b h_{\mathrm{eq} 1}^{3}}{L^{3} F_{0}}, \quad \bar{P}_{0}=\frac{P_{0}}{\left(F_{0} / b h_{\mathrm{eq} 1}\right)}
$$

3.1. Analysis of Dynamic Slip. Recalling the description in Olunloyo et al. [19], the relative slip at the interface of the sandwich smart structure is given by

$$
\Delta u(x, t)=u_{1}\left(x, t, 0^{-}\right)-u_{2}\left(x, t, 0^{+}\right),
$$

where $\xi$ is a dummy axial spatial variable of integration across the interface, $0^{+}$and $0^{-}$denote the origin of the transverse spatial variable for each layer, and $t$ is the time (state) variable.

Following Goodman and Klumpp [13], this can also be written as

$$
\begin{aligned}
\Delta u(x, t)= & E_{\text {eq } 1}^{-1} \int_{0}^{x}\left\{\left(\sigma_{x}\right)_{1}(\xi, 0, t, 0-)\right\} d \xi \\
& -E_{\text {eq } 2}^{-1} \int_{0}^{x}\left\{\left(\sigma_{x}\right)_{2}(\xi, 0, t, 0+)\right\} d \xi
\end{aligned}
$$


with the integral kernels as

$$
\begin{aligned}
\left(\sigma_{x}\right)_{1}\left(\xi, 0, t, 0^{-}\right)= & \frac{E_{\mathrm{eq} 1} h_{\mathrm{eq} 1}\left(C_{1}(\partial / \partial t)+1\right)}{2\left(1-v_{1}^{2}\right)} \\
& \times \frac{d^{2} W(\xi, t)}{d \xi^{2}}+\frac{\mu P_{a v}(x-L)}{h_{\mathrm{eq} 1}}, \\
\left(\sigma_{x}\right)_{2}\left(\xi, 0, t, 0^{+}\right)= & -\frac{E_{\mathrm{eq} 2} h_{\mathrm{eq} 2}\left(C_{1}(\partial / \partial t)+1\right)}{2\left(1-v_{1}^{2}\right)} \\
& \times \frac{d^{2} W(\xi, t)}{d \xi^{2}}-\frac{\mu P_{a v}(x-L)}{h_{\mathrm{eq} 2}},
\end{aligned}
$$

where

$$
\begin{aligned}
& h_{\mathrm{eq} 1}=\left(h_{1}+h_{a}\right), \quad h_{\mathrm{eq} 2}=\left(h_{2}+h_{s}\right), \\
& E_{\mathrm{eq} 1}=E_{1}\left(1+\gamma_{a, 1}\right), \quad E_{\mathrm{eq} 2}=E_{2}\left(1+\gamma_{s, 2}\right), \\
& \gamma_{\mathrm{eq}}=\frac{E_{\mathrm{eq} 1}}{E_{\mathrm{eq} 2}}=\frac{E_{1}\left(1+\gamma_{a, 1}\right)}{E_{2}\left(1+\gamma_{s, 2}\right)}, \quad \gamma_{a, 1}=\frac{E_{a}}{E_{1}}, \\
& \gamma_{s, 2}=\frac{E_{s}}{E_{2}}, \quad \Psi_{a, 1}=\frac{h_{a}}{h_{1}},
\end{aligned}
$$

$$
\Psi_{s, 2}=\frac{h_{s}}{h_{2}}, \quad \Psi_{a, 1}=\frac{h_{2}}{h_{1}}\left(\frac{1+\Psi_{s, 2}}{1+\Psi_{a, 1}}\right) .
$$

Equation (36) is integrated to give

$$
\begin{aligned}
\Delta \bar{u}= & \frac{1}{2}\left(\left(\frac{c_{1}}{\mathcal{A}}+\frac{c_{2} \Psi_{\mathrm{eq}}}{\mathscr{B}}\right) \frac{\partial}{\partial \tau}+\bar{\Delta}_{1}\right) \frac{\partial \bar{W}}{\partial \bar{x}} \\
& +\mu \bar{P}_{0} \bar{\Delta}_{2}\left(\bar{x}^{2}-2 \bar{x}\right)+\mu \bar{P}_{0} \varepsilon\left(\frac{2}{3} \bar{x}^{3}-\bar{x}^{2}\right),
\end{aligned}
$$

where the following have been introduced:

$$
\begin{aligned}
& \bar{\Delta}_{1}=\left(\frac{c_{1}}{2 \mathcal{A}}+\frac{c_{2} \Psi_{\mathrm{eq}}}{2 \mathscr{B}}\right), \\
& \bar{\Delta}_{2}=\left(\frac{1}{2 \mathcal{A}}+\frac{\Psi_{\mathrm{eq}}}{2 \mathscr{B}}\right) .
\end{aligned}
$$

Substitution for $\bar{W}$ from (29) simplifies (38) as

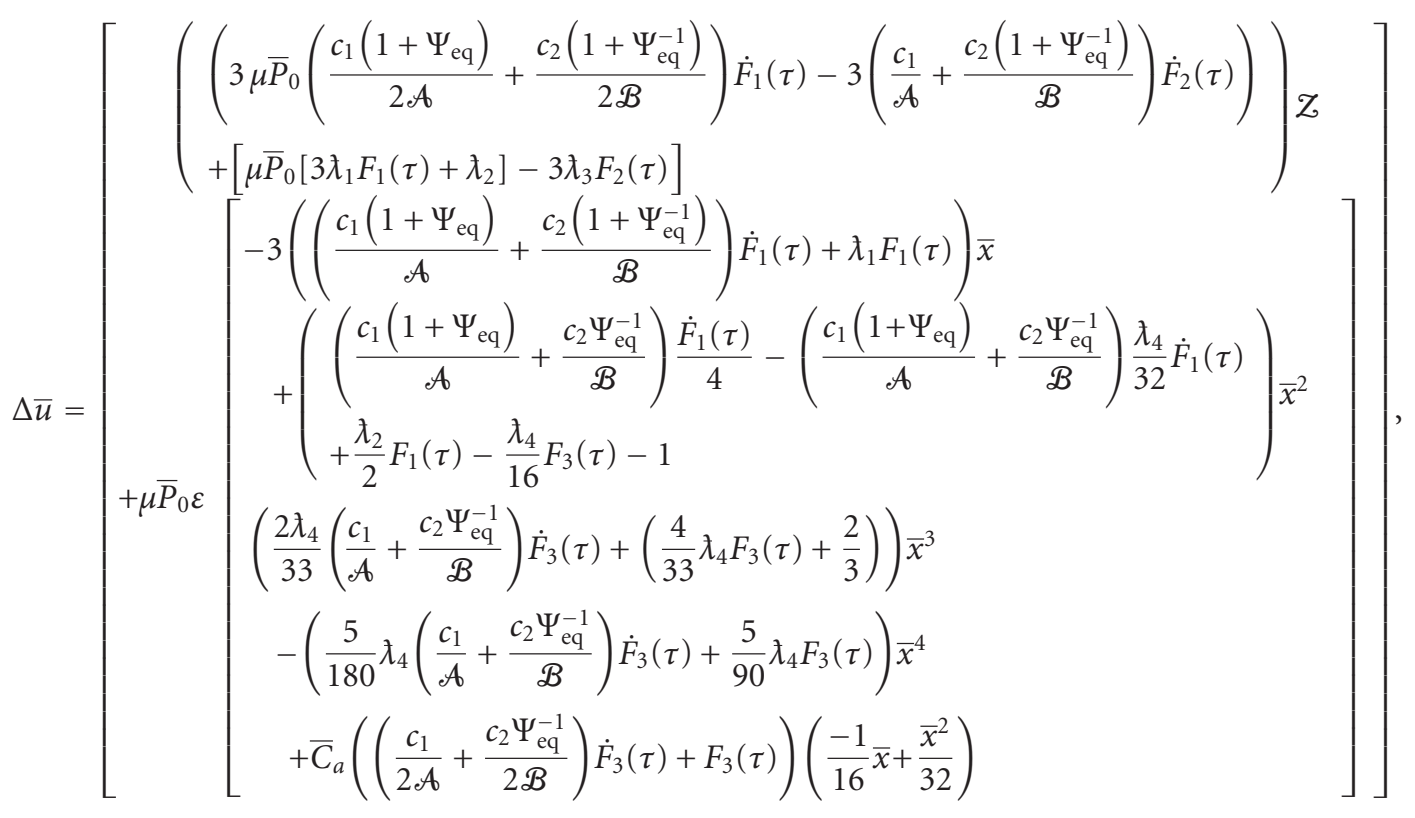

where $\mathcal{Z}$ denotes $\left(\bar{x}^{2}-2 \bar{x}\right)$

$$
\begin{aligned}
& \lambda_{1}=(\mathcal{C}) \cdot\left(\bar{\Delta}_{2}\right), \\
& \lambda_{2}=\left(\bar{\Delta}_{1}\right) \cdot\left(\bar{\Delta}_{2}\right), \quad \lambda_{3}=2 \cdot\left(\bar{\Delta}_{2}\right), \\
& \lambda_{4}=\left(\mathcal{A}+\mathcal{B} \gamma_{\mathrm{eq}}^{-1} \Psi_{\mathrm{eq}}^{-2}\right) .
\end{aligned}
$$

On setting $\varepsilon \rightarrow 0$ in (40), the dynamic slip at uniform pressure becomes

$$
\begin{aligned}
& \Delta \bar{u}
\end{aligned}
$$

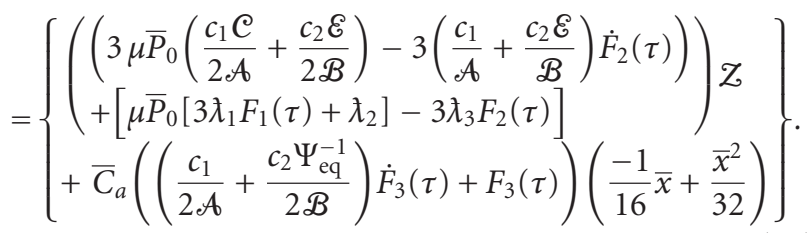

where $\mathcal{Z}$ denotes $\left(\bar{x}^{2}-2 \bar{x}\right), \mathcal{C}$ denotes $\left(1+\Psi_{\text {eq }}\right)$, and $\mathcal{E}$ denotes $\left(1+\Psi_{\text {eq }}^{-1}\right)$ 


\section{Energy Dissipation}

Following Goodman and Klumpp [13], the energy dissipated can be computed from the relation

$$
D=4 \mu b \int_{0}^{\pi / 2 \omega} \int_{0}^{L} P(x) \Delta u(x, t) d x d t
$$

and nondimensionalised as

$$
\bar{D}=4 \mu \int_{0}^{1 / 4} \int_{0}^{1} \bar{P}_{a v} \Delta \bar{u} d \bar{x} d \tau,
$$

where $\bar{D}=D(\bar{x}, \tau) E b h^{3} / L^{3} F_{0}^{2}$; substitution for $\Delta \bar{u}$ from (40) gives the closed form solution as

$$
\bar{D}=\bar{D}_{1}+\bar{D}_{2}+\bar{D}_{3}+\bar{D}_{4},
$$

where

$$
\bar{D}_{1}=\left[\begin{array}{c}
\left(8\left(\Delta_{5}(\eta) \lambda_{3}+\Delta_{3}(\eta)\left(\frac{c_{1}}{\mathcal{A}}+\frac{c_{2} \mathcal{E}}{\mathcal{B}}\right)\right)\right. \\
\left.-\left(\left(\frac{c_{1}}{\mathcal{A}}+\frac{c_{2} \mathcal{E}}{\mathcal{B}}\right) \Delta_{7}(\eta)+\Delta_{6}(\eta)\right) \frac{\bar{C}_{a}}{24}\right) \mu \bar{P}_{0} \\
-\left(\left(\frac{c_{1}}{\mathcal{A}}+\frac{c_{2} \mathcal{E}}{\mathcal{B}}\right)+\left(8 \lambda_{1} \Delta_{4}(\eta)+\frac{2}{3} \lambda_{2}\right)\right) \mu^{2} \bar{P}_{0}^{2}
\end{array}\right],
$$

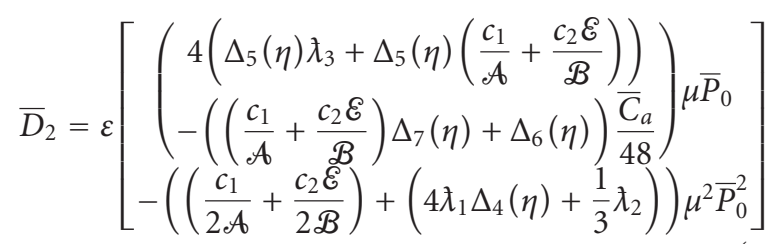

for all negative values of $\bar{C}_{a}$. Similarly,

$$
\begin{aligned}
& \left.\bar{D}_{3}=\left[\begin{array}{c}
\varepsilon\left(\left(\frac{c_{1}}{\mathcal{A}}+\frac{c_{2} \mathcal{E}}{\mathcal{B}}\right)\left(\left(\frac{y}{24}\right) \Delta_{8}(\eta)+\frac{41}{495} \lambda_{4} \Delta_{7}(\eta)\right)\right) \mu^{2} \bar{P}_{0}^{2} \\
+\varepsilon\left(\left(\frac{2 \lambda_{2}+6 \lambda_{1}}{3}\right) \Delta_{4}(\eta)+\frac{163}{1980} \lambda_{4} \Delta_{6}(\eta)-\frac{1}{6}\right) \mu^{2} \bar{P}_{0}^{2}
\end{array}\right)\right], \\
& \bar{D}_{4}=\left[\left(\begin{array}{c}
\varepsilon^{2}\left(\left(\frac{c_{1}}{\mathcal{A}}+\frac{c_{2} \mathcal{E}}{\mathcal{B}}\right)\left(\left(\frac{\mathcal{Y}}{48}\right) \Delta_{8}(\eta)+\frac{41}{990} \lambda_{4} \Delta_{7}(\eta)\right)\right) \mu^{2} \bar{P}_{0}^{2} \\
+\varepsilon^{2}\left(\left(\frac{\lambda_{2}+3 \lambda_{1}}{3}\right) \Delta_{4}(\eta)+\frac{163}{3960} \lambda_{4} \Delta_{6}(\eta)-\frac{1}{12}\right) \mu^{2} \bar{P}_{0}^{2}
\end{array}\right)\right],
\end{aligned}
$$

where

$$
\begin{aligned}
& \Delta_{3}(\eta)=\left[\frac{e^{-\pi \bar{\kappa}_{1} / 2}}{\overline{\mathcal{K}}_{2} \overline{\mathcal{K}}_{3}}+\frac{e^{-\pi \bar{\kappa}_{4} / 2}}{\bar{\kappa}_{3} \bar{\kappa}_{5}}+\frac{e^{i \pi \eta / 2}}{\overline{\mathcal{K}}_{6} \overline{\mathcal{K}}_{7}}+\frac{\omega^{-1} \overline{\mathcal{K}}_{11} e^{i \pi \eta / 2}}{\overline{\mathcal{K}}_{9} \overline{\mathcal{K}}_{10}}\right], \\
& \Delta_{4}(\eta)=\left[\left(\frac{(\mathcal{A B})}{4 \mathscr{B}+\gamma_{\mathrm{eq}} \Psi_{\mathrm{eq}}^{3} \mathscr{A}}\right)\right. \\
& \left.-\frac{e^{-\pi \bar{\kappa}_{1} / 2}}{2 \pi \bar{\kappa}_{1}^{2} \bar{\kappa}_{2} \bar{\kappa}_{12}}+\frac{e^{-\pi \bar{\kappa}_{1} / 2}}{2 \pi \bar{\kappa}_{1} \bar{\kappa}_{4} \bar{\kappa}_{5} \overline{\mathcal{\kappa}}_{12}}+\frac{\omega^{-1} e^{-\pi \eta \bar{\kappa}_{1} / 2}}{2 \pi \bar{\kappa}_{1} \bar{\kappa}_{13}}\right], \\
& \Delta_{5}(\eta)=\left[\frac{e^{-\pi \bar{\kappa}_{1} / 2}}{2 \pi \bar{\kappa}_{1} \bar{\kappa}_{2} \bar{\kappa}_{3}}+\frac{e^{-\pi \bar{\kappa}_{4} / 2}}{2 \pi \overline{\mathcal{K}}_{3} \overline{\mathcal{K}}_{4} \overline{\mathcal{K}}_{5}}-i \frac{e^{i \pi \eta / 2}}{2 \pi \eta \overline{\mathcal{K}}_{6} \overline{\mathcal{K}}_{7}}-i \frac{\omega^{-1} \overline{\mathcal{K}}_{11} e^{i \pi \eta / 2}}{2 \pi \eta \overline{\mathcal{K}}_{9} \overline{\mathcal{K}}_{10}}\right], \\
& \Delta_{6}(\eta)=\left[\frac{1}{4}-\frac{e^{-\pi \bar{\kappa}_{1} / 2}}{2 \pi \bar{\kappa}_{1}^{2} \bar{\kappa}_{12}}-\frac{e^{-\pi \bar{\kappa}_{4} / 2}}{2 \pi \bar{\kappa}_{4}^{2} \bar{\kappa}_{12}}\right] \text {, } \\
& \Delta_{7}(\eta)=\left[1+\frac{e^{-\pi \bar{\kappa}_{1} / 2}}{\overline{\mathcal{K}}_{1} \bar{\kappa}_{12}}+\frac{e^{-\pi \bar{\kappa}_{4} / 2}}{\overline{\mathcal{\kappa}}_{4} \bar{\kappa}_{12}}\right], \\
& \Delta_{8}(\eta)=\left[\left(\frac{(\mathscr{A} \mathscr{B})}{\mathscr{B}+\gamma_{\mathrm{eq}} \Psi_{\mathrm{eq}}^{3} \mathcal{A}}\right)\right. \\
& \left.+\frac{e^{-\pi \bar{\kappa}_{1} / 2}}{\overline{\mathcal{K}}_{1} \overline{\mathcal{K}}_{2} \overline{\mathcal{K}}_{12}}-\frac{e^{-\pi \bar{\kappa}_{1} / 2}}{\overline{\mathcal{K}}_{4} \overline{\mathcal{K}}_{5} \overline{\mathcal{K}}_{12}}-\frac{\omega^{-1} e^{-\pi \eta \bar{\kappa}_{1} / 2}}{\overline{\mathcal{\kappa}}_{13}}\right] .
\end{aligned}
$$

\section{Analysis of Logarithmic Damping Decrement}

For this problem the logarithmic damping coefficient is used as a measure of the damping capacity of the structure under consideration. Following Masuko et al. [24], the relationship between the energy dissipation from two consecutive cycles and the associated logarithmic damping decrement satisfies the form

$$
\delta=\frac{1}{2} \ln \left(\frac{E_{n}}{E_{n+1}}\right)=\frac{1}{2} \ln \left(\frac{E_{\mathrm{ne}}+E_{\text {loss }}}{E_{n} E_{\text {loss }}}\right),
$$

where $E_{\mathrm{ne}}$ is the strain energy of the laminate material, whereas $E_{\text {loss }}$ is the energy loss per cycle, and $\delta$ is the Logarithmic damping decrement.

In particular, $E_{\text {loss }}$, the slip energy, is computed from (45)-(46a), (46b), (46c), and (46d), that is $\bar{D}$, whereas $E_{\text {ne }}$ is presently analyzed.

5.1. Analysis of Strain Energy of the Clamped Smart Structure. The total strain energy of the smart structure is a combination of the energy introduced by the bending moment as well and that stored from the deflection of the free end. 
The energy from the bending moment can be evaluated from Castigliano's theorem, namely,

$$
U_{1}=\int_{0}^{L} \frac{M^{2}}{2 E_{\mathrm{eq}} I_{\mathrm{eq}}} d x,
$$

whilst the energy stored at the free end follows from the theory of strength of materials namely,

$$
U_{2}=\frac{3}{2} \frac{E_{\mathrm{eq}}}{L^{3}} W_{L}^{2}
$$

here, $M$ is the bending moment, $E_{\mathrm{eq}}$ the modulus of rigidity of the material, $I_{\mathrm{eq}}=(2 / 3) b h_{\mathrm{eq}}^{3}$ the moment of inertia of the clamped sandwich composite smart structure, and $W_{L}$ the deflection at the free end.

For this case, the computed nondimensionalized strain energy components are

$$
\bar{U}_{1}=27\left\{\begin{array}{l}
\Theta_{1} e^{i 4 \pi \eta}-\Theta_{2} e^{2 \pi \eta(i-\chi)}+\omega^{-2} \Theta_{3} e^{-4 \pi \chi \eta} \\
-2 \mu \bar{P}_{0}\left(\left(1+\frac{\varepsilon}{2}\right) \mathcal{C}\right)\left(\Theta_{4} e^{i 2 \pi \eta}-\Theta_{5} \omega^{-1} e^{2 \pi \eta(i-\chi)}-\Theta_{6} \omega^{-1} e^{-2 \pi \chi \eta}+\Theta_{7} \omega^{-2} e^{-4 \pi \chi \eta}\right) \\
+\mu^{2} \bar{P}_{0}^{2}\left(\left(1+\varepsilon+\frac{\varepsilon^{2}}{4}\right) e^{2}\right)\left(\Theta_{8}-2 \Theta_{9} \omega^{-1} e^{-2 \pi \chi \eta}+\Theta_{10} \omega^{-2} e^{-4 \pi \chi \eta}\right)
\end{array}\right\},
$$

where

$$
\begin{aligned}
& \Theta_{1}=\left(\frac{2 \mathcal{A} \mathscr{B}}{\left(\mathscr{B}\left(1+\mu_{1}\right)+\mathcal{A}\left(1+\mu_{2}\right) \gamma_{\mathrm{eq}} \Psi_{\mathrm{eq}}\right)}\right)^{2} \\
& \Theta_{2}=4\left(\frac{\mathcal{A B}}{\left(\mathscr{B}+\mathcal{A} \gamma_{\mathrm{eq}} \Psi_{\mathrm{eq}}^{3}-i\left(\mu_{1} \mathcal{B}+\mu_{2} \mathcal{B} \gamma_{\mathrm{eq}} \Psi_{\mathrm{eq}}^{3}\right)\right)}\right), \\
& \Theta_{3}=\left(\frac{\mu_{1} \mathcal{B}+\mu_{2} \mathcal{A} \gamma_{\mathrm{eq}} \Psi_{\mathrm{eq}}^{3}}{\left(\mathcal{B}+\mathcal{A} \gamma_{\mathrm{eq}} \Psi_{\mathrm{eq}}^{3}-i\left(\mu_{1} \mathcal{B}+\mu_{2} \mathcal{B} \gamma_{\mathrm{eq}} \Psi_{\mathrm{eq}}^{3}\right)\right)}\right)^{2} \\
& \Theta_{4}=\left(\frac{(\mathcal{A B})^{2}}{\left(\mathscr{B}\left(1+\bar{\mu}_{1}\right)+\mathcal{A}\left(1+\bar{\mu}_{2}\right) \gamma_{\mathrm{eq}} \Psi_{\mathrm{eq}}^{3}\right)\left(\mathscr{B}+\mathcal{A} \gamma_{\mathrm{eq}} \Psi_{\mathrm{eq}}^{3}\right)}\right)^{2}, \\
& \Theta_{5}=\left(\frac{\mathcal{A B}}{\left(\mathscr{B}+\mathcal{A} \gamma_{\mathrm{eq}} \Psi_{\mathrm{eq}}^{3}\right)}\right) \text {, } \\
& \Theta_{6}=\left(\left(\frac{\mathcal{A} \mathscr{B}}{\left(\mathscr{B}+\mathcal{A} \gamma_{\mathrm{eq}} \Psi_{\mathrm{eq}}^{3}\right)}\right)\right. \\
& \left.\times\left(\frac{\bar{\mu}_{1} \mathcal{B}+\bar{\mu}_{2} \mathcal{A} \gamma_{\mathrm{eq}} \Psi_{\mathrm{eq}}^{3}}{\left(\mathcal{B}+\mathcal{A} \gamma_{\mathrm{eq}} \Psi_{\mathrm{eq}}^{3}-i\left(\bar{\mu}_{1} \mathcal{B}+\bar{\mu}_{2} \mathcal{B} \gamma_{\mathrm{eq}} \Psi_{\mathrm{eq}}^{3}\right)\right)}\right)\right), \\
& \Theta_{7}=\left(\frac{\left(\bar{\mu}_{1} \mathcal{B}+\bar{\mu}_{2} \mathcal{A} \gamma_{\mathrm{eq}} \Psi_{\mathrm{eq}}^{3}\right)^{2}}{\left(\mathcal{B}+\mathcal{A} \gamma_{\mathrm{eq}} \Psi_{\mathrm{eq}}^{3}\right)\left(\mathcal{B}+\mathcal{A} \gamma_{\mathrm{eq}} \Psi_{\mathrm{eq}}^{3}-i\left(\bar{\mu}_{1} \mathcal{B}+\bar{\mu}_{2} \mathcal{B} \gamma_{\mathrm{eq}} \Psi_{\mathrm{eq}}^{3}\right)\right)}\right) \text {, } \\
& \Theta_{8}=\left(\frac{\mathcal{A} B}{\mathcal{B}+\mathcal{A} \gamma_{\mathrm{eq}} \Psi_{\mathrm{eq}}^{3}}\right)^{2} \text {, } \\
& \Theta_{9}=\left(\frac{\left(\left(1-v_{1}\right)\left(1-v_{2}\right)\right)\left(\bar{\mu}_{1} \mathcal{B}+\bar{\mu}_{2} \mathcal{A} \gamma_{\mathrm{eq}} \Psi_{\mathrm{eq}}^{3}\right)}{\left(\mathcal{B}+\mathcal{A} \gamma_{\mathrm{eq}} \Psi_{\mathrm{eq}}^{3}\right)^{2}}\right) \text {, }
\end{aligned}
$$




$$
\begin{aligned}
& \Theta_{10}=\left(\frac{\bar{\mu}_{1} \mathcal{B}+\bar{\mu}_{2} \mathcal{A} \gamma_{\mathrm{eq}} \Psi_{\mathrm{eq}}^{3}}{\left(\mathcal{B}+\mathcal{A} \gamma_{\mathrm{eq}} \Psi_{\mathrm{eq}}^{3}\right)}\right)^{2}, \\
& \chi=\left(\frac{\bar{\mu}_{1} \mathcal{B}+\bar{\mu}_{2} \mathcal{A} \gamma_{\mathrm{eq}} \Psi_{\mathrm{eq}}^{3}}{\left(\mathcal{B}+\mathcal{A} \gamma_{\mathrm{eq}} \Psi_{\mathrm{eq}}^{3}\right)}\right) \text {, } \\
& \bar{U}_{2}=\left\{\begin{array}{l}
\Theta_{1} e^{i 4 \pi \eta}-\Theta_{2} e^{2 \pi \eta(i-\chi)}+\omega^{-2} \Theta_{3} e^{-4 \pi \chi \eta} \\
-2 \mu \bar{P}_{0}\left(\left(1+\frac{\varepsilon}{2}\right) \mathcal{C}\right)\left(\Theta_{4} e^{i 2 \pi \eta}-\Theta_{5} \omega^{-1} e^{2 \pi \eta(i-\chi)}-\Theta_{6} \omega^{-1} e^{-2 \pi \chi \eta}+\Theta_{7} \omega^{-2} e^{-4 \pi \chi \eta}\right) \\
+\mu^{2} \bar{P}_{0}^{2}\left(\left(1+\varepsilon+\frac{\varepsilon^{2}}{4}\right) e^{2}\right)\left(\Theta_{8}-2 \Theta_{9} \omega^{-1} e^{-2 \pi \chi \eta}+\Theta_{10} \omega^{-2} e^{-4 \pi \chi \eta}\right) \\
\\
{\left[\begin{array}{l}
\left(\frac{\Theta_{11}}{480} \mu \bar{P}_{0} \varepsilon+\frac{\Theta_{15}}{48} \bar{C}_{a}\right) e^{i 2 \pi \eta}-\omega^{-1}\left(\frac{\Theta_{12}}{480} \mu \bar{P}_{0} \varepsilon+\frac{\Theta_{16}}{48} \bar{C}_{a}\right) e^{-2 \pi \chi \eta} \\
-\frac{\Theta_{13}}{480} \mu^{2} \bar{P}_{0}^{2}\left(\varepsilon+\frac{\varepsilon^{2}}{2}\right) \mathcal{C}+\omega^{-1} \frac{\Theta_{14}}{480} \mu^{2} \bar{P}_{0}^{2}\left(\varepsilon+\frac{\varepsilon^{2}}{2}\right) \mathcal{e} e^{-2 \pi \chi \eta} \\
-\frac{1}{96} \mu \bar{P}_{0}\left(1+\frac{\varepsilon}{2}\right) \mathcal{C} \bar{C}_{a}\left(\Theta_{17}-\omega^{-1} \Theta_{18} e^{-2 \pi \chi \eta}\right) \\
+
\end{array}\right], \frac{\Theta_{19}}{960^{2}} \mu^{2} \bar{P}_{0}^{2} \varepsilon^{2}+\frac{\Theta_{20}}{96^{2}} \mu \bar{P}_{0} \varepsilon \bar{C}_{a}+\frac{1}{96^{2}} \bar{C}_{a}^{2}}
\end{array}\right],
\end{aligned}
$$

while

$$
\begin{aligned}
& \Theta_{11}=\frac{2 \mathscr{A B}\left(\mathcal{A}+\mathscr{B} \gamma_{\mathrm{eq}}^{-1} \Psi_{\mathrm{eq}}^{-2}\right)}{\left(\mathscr{B}\left(1+\bar{\mu}_{1}\right)+\mathcal{A}\left(1+\bar{\mu}_{2}\right) \gamma_{\mathrm{eq}} \Psi_{\mathrm{eq}}^{3}\right)}, \\
& \Theta_{12}=\left(\frac{2\left(\mathcal{A}+\mathscr{B} \gamma_{\mathrm{eq}}^{-1} \Psi_{\mathrm{eq}}^{-2}\right)\left(\bar{\mu}_{1} \mathcal{B}+\bar{\mu}_{2} \mathcal{A} \gamma_{\mathrm{eq}} \Psi_{\mathrm{eq}}^{3}\right)}{\left(\mathcal{B}+\mathcal{A} \gamma_{\mathrm{eq}} \Psi_{\mathrm{eq}}^{3}\right)\left(\mathcal{B}+\mathcal{A} \gamma_{\mathrm{eq}} \Psi_{\mathrm{eq}}^{3}-i\left(\bar{\mu}_{1} \mathcal{B}+\bar{\mu}_{2} \mathcal{B} \gamma_{\mathrm{eq}} \Psi_{\mathrm{eq}}^{3}\right)\right)}\right), \\
& \Theta_{13}=\frac{\mathcal{A} \mathcal{B}\left(\mathcal{A}+\mathscr{B} \gamma_{\mathrm{eq}}^{-1} \Psi_{\mathrm{eq}}^{-2}\right)}{\left(\mathscr{B}+\mathcal{A} \gamma_{\mathrm{eq}} \Psi_{\mathrm{eq}}^{3}\right)} \\
& \Theta_{14}=\frac{\left(\mathscr{B} \bar{\mu}_{1}+\mathcal{A} \bar{\mu}_{2} \gamma_{\mathrm{eq}} \Psi_{\mathrm{eq}}^{3}\right)\left(\mathcal{A} \mathcal{B} \gamma_{\mathrm{eq}}^{-1} \Psi_{\mathrm{eq}}^{-2}\right)}{\left(\mathcal{B}+\mathcal{A} \gamma_{\mathrm{eq}} \Psi_{\mathrm{eq}}^{3}\right)} \text {, } \\
& \Theta_{15}=\frac{\mathcal{A B}}{\left(\mathscr{B}\left(1+\bar{\mu}_{1}\right)+\mathcal{A}\left(1+\bar{\mu}_{2}\right) \gamma_{\mathrm{eq}} \Psi_{\mathrm{eq}}^{3}\right)} \text {, } \\
& \Theta_{16}=\left(\frac{\left(\bar{\mu}_{1} \mathcal{B}+\bar{\mu}_{2} \mathcal{A} \gamma_{\mathrm{eq}} \Psi_{\mathrm{eq}}^{3}\right)}{\left(\mathcal{B}+\mathcal{A} \gamma_{\mathrm{eq}} \Psi_{\mathrm{eq}}^{3}-i\left(\bar{\mu}_{1} \mathcal{B}+\bar{\mu}_{2} \mathcal{B} \gamma_{\mathrm{eq}} \Psi_{\mathrm{eq}}^{3}\right)\right)}\right) \text {, } \\
& \Theta_{17}=\frac{2 \mathcal{A} \mathscr{B}}{\left(\mathscr{B}+\mathcal{A} \gamma_{\mathrm{eq}} \Psi_{\mathrm{eq}}^{3}\right)}, \\
& \Theta_{18}=\frac{2\left(\mathcal{B} \bar{\mu}_{1}+\mathcal{A} \bar{\mu}_{2} \gamma_{\mathrm{eq}} \Psi_{\mathrm{eq}}^{3}\right)}{\left(\mathcal{B}+\mathcal{A} \gamma_{\mathrm{eq}} \Psi_{\mathrm{eq}}^{3}\right)}, \\
& \Theta_{19}=\left(\mathcal{A}+\mathscr{B} \gamma_{\mathrm{eq}}^{-1} \Psi_{\mathrm{eq}}^{-2}\right)^{2} \text {, } \\
& \Theta_{20}=\frac{\left(\mathcal{A}+\mathcal{B} \gamma_{\mathrm{eq}}^{-1} \Psi_{\mathrm{eq}}^{-2}\right)^{2}}{5} \text {. }
\end{aligned}
$$


Here, we have introduced the nondimensionalization

$$
\bar{U}=\frac{U_{n} E b h^{3}}{F_{0}^{2} L^{3}}, \quad n=1,2,
$$

to write the logarithmic damping decrement as

$$
\delta=\frac{1}{2} \ln \left(1+\frac{\overline{\mathrm{D}}}{\overline{U_{1}}+\overline{U_{2}}}\right) .
$$

\section{Analysis of Spatial Transfer Functions for Active Vibration and Noise Control Designs}

Several authors in the field of active noise and vibration control have derived useful rational expressions with experimental verification for transfer functions with piezoelectric beams or smart structures systems such as in $[12,25-$ 30]. These transfer functions are characterized by a common denominator that is strongly influenced by the parameters of the piezoelectric beams or smart structures. For this paper, the focus is to derive closed-form analytic rational expressions in the Laplace domain for both the active control of the dynamic deflection and interfacial slip at the interface of the layered sandwich composite elastic beam-plate smart structure. We shall now proceed to address these issues.

6.1. Computation of Spatial Transfer Functions for Active Control of Dynamic Deflection and Interfacial Slip. The transfer function for the active control of dynamic deflection can be derived or computed from (28) as

$$
\begin{aligned}
\frac{\widetilde{W}(\bar{x}, s)}{\widetilde{F}(s)}=\frac{\widetilde{W}(\bar{x}, s) L^{3}}{E_{\mathrm{eq} 1} b h_{\mathrm{eq} 1}^{3}}=T F_{1} \\
=\frac{L^{3}\left(\begin{array}{l}
\left(2 \widetilde{F}_{2}(s)-\mu \bar{P}_{0} \mathcal{C} \widetilde{F}_{1}(s)\right)\left(3 \bar{x}^{2}-\bar{x}^{3}\right) \\
+\mu \bar{P}_{0}\left[-\frac{3 \mathcal{C}}{2} \widetilde{F}_{1}(s) x^{2}+\left(\left(\frac{\mathcal{C}}{2}\right) \widetilde{F}_{1}(s)-\left(\frac{\mathcal{A}+\mathcal{B} \gamma_{\mathrm{eq}}^{-1} \Psi_{\mathrm{eq}}^{-2}}{48}\right) \widetilde{F}_{3}(s)\right) x^{3}\right] \varepsilon \\
+\widetilde{F}_{3}(s)\left[\left(\mathcal{A}+\mathcal{B} \gamma_{\mathrm{eq}}^{-1} \Psi_{\mathrm{eq}}^{-2}\right)\left(\frac{x^{4}}{33}-\frac{x^{5}}{90}\right)\right]+\bar{C}_{a} \widetilde{F}_{1}(s)\left(\frac{-\bar{x}^{2}}{32}+\frac{\bar{x}^{3}}{96}\right)
\end{array}\right)}{E_{\mathrm{eq} 1} b h_{\mathrm{eq} 1}^{3}},
\end{aligned}
$$

while the corresponding transfer function for the active control of dynamic interfacial slip can be derived or computed from (40) as

$$
\begin{aligned}
& \frac{\Delta \tilde{u}(\bar{x}, s)}{\widetilde{F}(s)}=\frac{\Delta \bar{u}(\bar{x}, s) L^{2}}{E_{\text {eq } 1} b h_{\mathrm{eq} 1}^{2}}=T F_{2}
\end{aligned}
$$

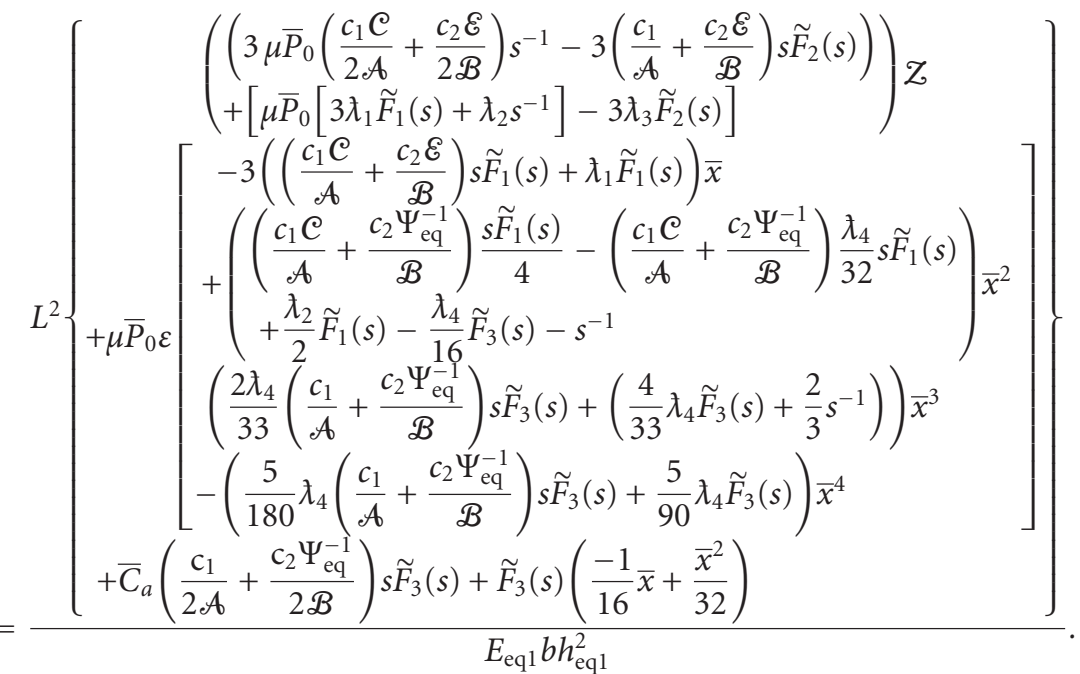


Within the context of robust active vibration and noise control designs, the common denominator admits the form

$$
\begin{aligned}
& \widetilde{\widetilde{W}}(\bar{x}, s) \\
& =\left(\begin{array}{l}
\left(2 \widetilde{F}_{2}(s)-\mu \bar{P}_{0} \mathcal{C} \widetilde{F}_{1}(s)\right)\left(3 \bar{x}^{2}-\bar{x}^{3}\right) \\
+\mu \bar{P}_{0}\left[-\frac{3 \mathcal{C}}{2} \widetilde{F}_{1}(s) x^{2}+\left(\left(\frac{\mathcal{C}}{2}\right) \widetilde{F}_{1}(s)-\left(\frac{\mathcal{A}+\mathscr{B} \gamma_{\mathrm{eq}}^{-1} \Psi_{\mathrm{eq}}^{-2}}{48}\right) \widetilde{F}_{3}(s)\right) x^{3}\right] \\
+\widetilde{F}_{3}(s)\left[\left(\mathcal{A}+\mathcal{B} \gamma_{\mathrm{eq}}^{-1} \Psi_{\mathrm{eq}}^{-2}\right)\left(\frac{x^{4}}{33}-\frac{x^{5}}{90}\right)\right]+\bar{C}_{a} \widetilde{F}_{1}(s)\left(\frac{-\bar{x}^{2}}{32}+\frac{\bar{x}^{3}}{96}\right)
\end{array}\right),
\end{aligned}
$$

where $\widetilde{F}_{1}(s), \widetilde{F}_{2}(s)$, and $\widetilde{F}_{3}(s)$ are the Laplace transform of $F_{1}(\tau), F_{2}(\tau)$ and $F_{3}(\tau)$ as previously defined, subject to zero initial conditions, namely, $F_{1}(0), F_{2}(0)$, and $F_{3}(0)$.

\section{Analysis of Results}

By studying Figure 2, it is significant to note that for this case $\varepsilon=0$ and irrespective of the value of the piezoelectric parameter $\bar{C}_{a}$, the energy dissipation assumes the same parabolic profile reported in earlier studies such as Damisa et al. $[15,16]$ and Olunloyo et al. $[19,20]$. This is a direct consequence of (45) and ((46a)-(46d)) from which we deduce the condition for optimal energy dissipation as

$$
\begin{aligned}
\mu \bar{P}_{\mathrm{opt}}=(2+\mathcal{E}) \Gamma^{-1}( & 4\left(\Delta_{5}(\eta) \lambda_{3}+\Delta_{3}(\eta)\left(\frac{c_{1}}{\mathcal{A}}+\frac{c_{2} \mathscr{E}}{\mathscr{B}}\right)\right) \\
& \left.-\left(\left(\frac{c_{1}}{\mathcal{A}}+\frac{c_{2} \mathscr{E}}{\mathscr{B}}\right) \Delta_{7}(\eta)+\Delta_{6}(\eta)\right) \frac{\bar{C}_{a}}{48}\right),
\end{aligned}
$$

where

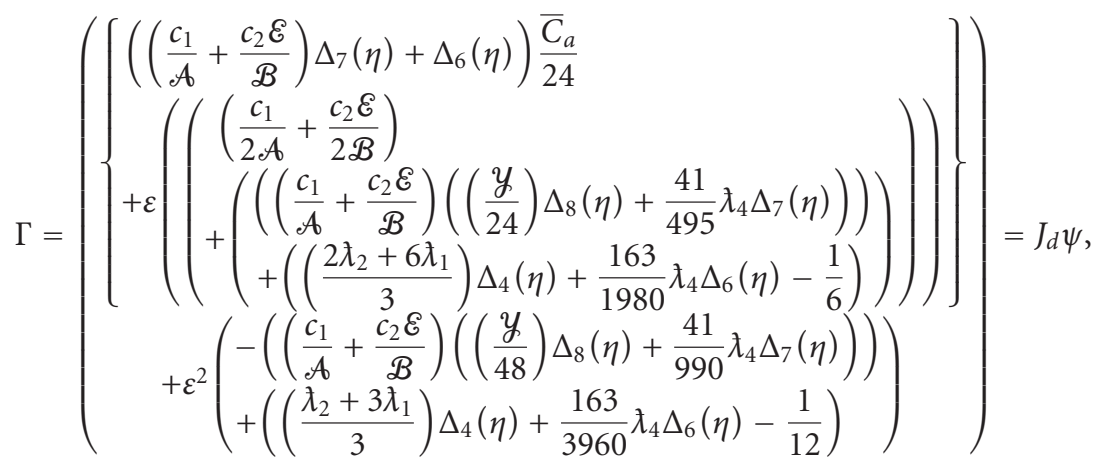

where $y$ denotes $-144-7 \lambda_{4}$

$$
\begin{gathered}
J_{d}=(2+\varepsilon) \Gamma^{-1}, \\
\psi=\left(\begin{array}{c}
4\left(\Delta_{5}(\eta) \lambda_{3}+\Delta_{3}(\eta)\left(\frac{c_{1}}{\mathcal{A}}+\frac{c_{2} \mathcal{E}}{\mathcal{B}}\right)\right) \\
-\left(\left(\frac{c_{1}}{\mathcal{A}}+\frac{c_{2} \mathcal{E}}{\mathscr{B}}\right) \Delta_{7}(\eta)+\Delta_{6}(\eta)\right) \frac{\bar{C}_{a}}{48}
\end{array}\right)
\end{gathered}
$$

The use of (59) gives the optimal slip energy as

$$
\bar{D}_{\mathrm{opt}}=\sum_{n=1}^{4} \bar{D}_{n_{\mathrm{opt}}},
$$

where

$$
\begin{gathered}
\bar{D}_{\text {lopt }}=J_{d}\left[\left(2 \psi^{2}\right)-J_{d} \psi\left(\frac{c_{1}}{\mathcal{A}}+\frac{c_{2} \mathcal{E}}{\mathcal{B}}\right)+\left(8 \lambda_{1} \Delta_{4}(\eta)+\frac{2}{3} \lambda_{2}\right)\right] \\
\bar{D}_{2 \mathrm{opt}}=J_{d} \varepsilon\left[\left(\psi^{2}\right)-J_{d} \psi\left(\left(\frac{c_{1}}{2 \mathcal{A}}+\frac{c_{2} \mathcal{E}}{2 \mathscr{B}}\right)+\left(4 \lambda_{1} \Delta_{4}(\eta)+\frac{1}{3} \lambda_{2}\right)\right)\right], \\
\bar{D}_{3 \mathrm{opt}}=J_{d}^{2} \psi^{2}\left[\varepsilon\left(\left(\frac{c_{1}}{\mathcal{A}}+\frac{c_{2} \mathcal{E}}{\mathcal{B}}\right)\left(\begin{array}{c}
\left(\frac{\mathcal{y}}{24}\right) \Delta_{8}(\eta) \\
+\frac{41}{495} \lambda_{4} \Delta_{7}(\eta)
\end{array}\right)\right)\right. \\
+\varepsilon\left(\begin{array}{c}
\left(\frac{2 \lambda_{2}+6 \lambda_{1}}{3}\right) \Delta_{4}(\eta) \\
\left.\left.+\frac{163}{1980} \lambda_{4} \Delta_{6}(\eta)-\frac{1}{6}\right)\right],
\end{array}\right.
\end{gathered}
$$




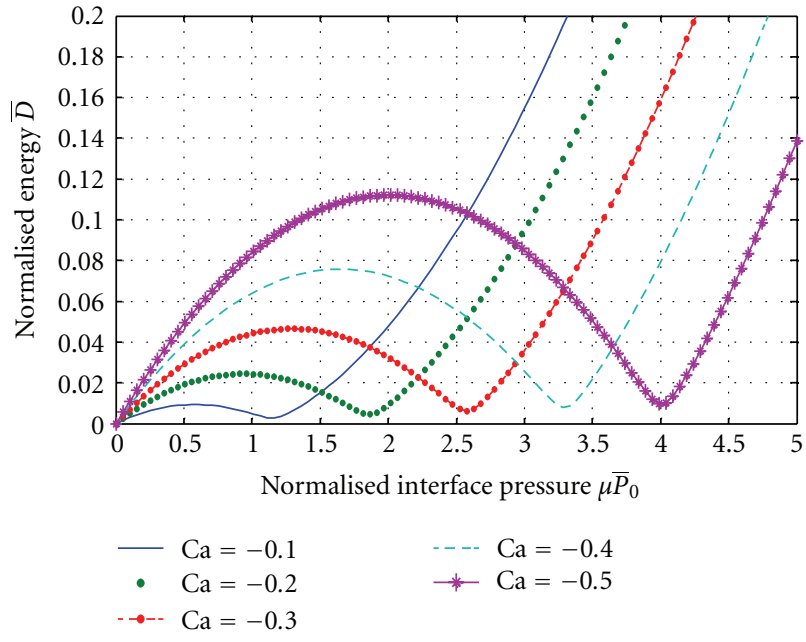

FIGURE 4: Energy dissipation profile for the case $\varepsilon=0 ; v_{1}=$ $0.35, v_{2}=0.25, \gamma_{\mathrm{eq}}=0.1, \Psi_{\mathrm{eq}}=0.001, \bar{\mu}_{1}=0.4, \bar{\mu}_{2}=0.2$, and frequency ratio $\eta=0.005$ and $\omega=8 \mathrm{KHz}$.

$$
\begin{gathered}
\bar{D}_{4 \mathrm{opt}}=J_{d}^{2} \psi^{2}\left[\left(\mathcal{E}^{2}\left(\left(\frac{c_{1}}{\mathcal{A}}+\frac{c_{2} \mathcal{E}}{\mathcal{B}}\right)\left(\left(\frac{\mathcal{y}}{48}\right) \Delta_{8}(\eta)+\frac{41}{990} \lambda_{4} \Delta_{7}(\eta)\right)\right)\right.\right. \\
\left.\left.+\varepsilon^{2}\left(\begin{array}{c}
\left(\frac{\lambda_{2}+3 \lambda_{1}}{3}\right) \Delta_{4}(\eta) \\
+\frac{163}{3960} \lambda_{4} \Delta_{6}(\eta)-\frac{1}{12}
\end{array}\right)\right)\right]
\end{gathered}
$$

This result highlights the effects of the piezoelectric parameter $\bar{C}_{a}$ and the associated frequency ratio $\kappa$ as embedded in the parameter $\bar{\Delta}(\kappa)$ with selected values of $\gamma_{\mathrm{eq}}, \Psi_{\mathrm{eq}}$, and Poisson's ratios $v_{1}, v_{2}$ of the composite elastic beam-plate layers (1 and 2). Relation (59) also shows that in the absence of interfacial pressure gradient, the excitation frequency has a significant role to play in defining the optimal pressure for slip damping. In fact a comparison of Figure 4 with Figure 6 or Figure 5 with Figure 7 illustrates that higher frequency ratios support higher energy dissipation as expected with viscoelastic structures. In all cases, the level of dissipation increases with the value of the piezoelectric parameter $\bar{C}_{a}$ thereby confirming the efficacy of the introduction of smart structures into the damping mechanism.

Also of interest here is to ascertain to what extent can we influence the amount of energy dissipated either by our choice of different materials for the upper and lower laminates or by varying the thicknesses of the upper and lower laminates? These effects are best simulated by varying the values of $\gamma_{\mathrm{eq}}$ and $\Psi_{\mathrm{eq}}$, these being the relative ratios of Young's moduli and laminate thicknesses, respectively,

$$
\begin{gathered}
{\left[\gamma_{\mathrm{eq}}=\frac{E_{2}}{E_{1}}\left(\frac{1+\gamma_{s, 2}}{1+\gamma_{a, 1}}\right), \gamma_{s, 2}=\frac{E_{s}}{E_{2}}, \gamma_{a, 1}=\frac{E_{a}}{E_{1}},\right.} \\
\left.\Psi_{\mathrm{eq}}=\frac{h_{2}}{h_{1}}\left(\frac{1+\Psi_{s, 2}}{1+\Psi_{a, 1}}\right), \Psi_{s, 2}=\frac{h_{s}}{h_{2}}, \Psi_{a, 1}=\frac{h_{a}}{h_{1}}\right] .
\end{gathered}
$$

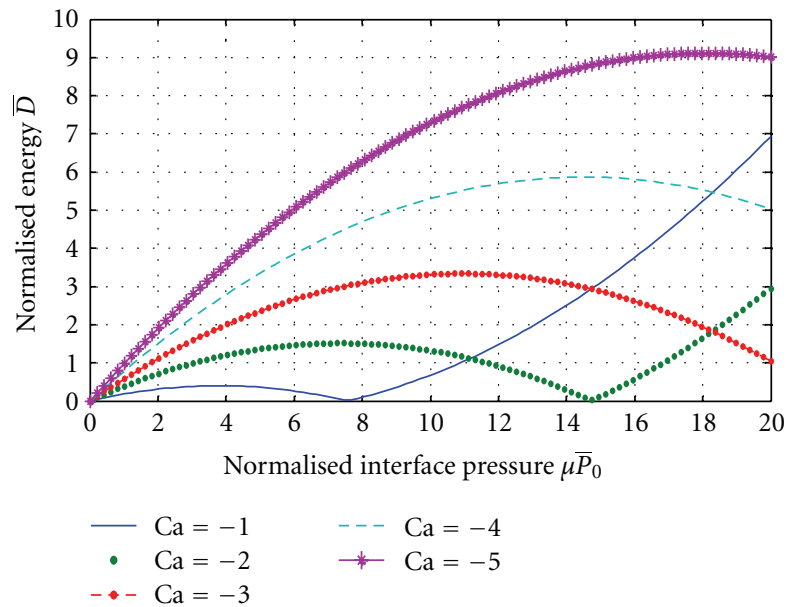

FIGURE 5: Energy dissipation profile for the case $\varepsilon=0$; $v_{1}=$ $0.35, v_{2}=0.25, \gamma_{\mathrm{eq}}=0.1, \Psi_{\mathrm{eq}}=0.001, \bar{\mu}_{1}=0.4, \bar{\mu}_{2}=0.2$, and frequency ratio $\eta=0.005$ and $\omega=8 \mathrm{KHz}$.

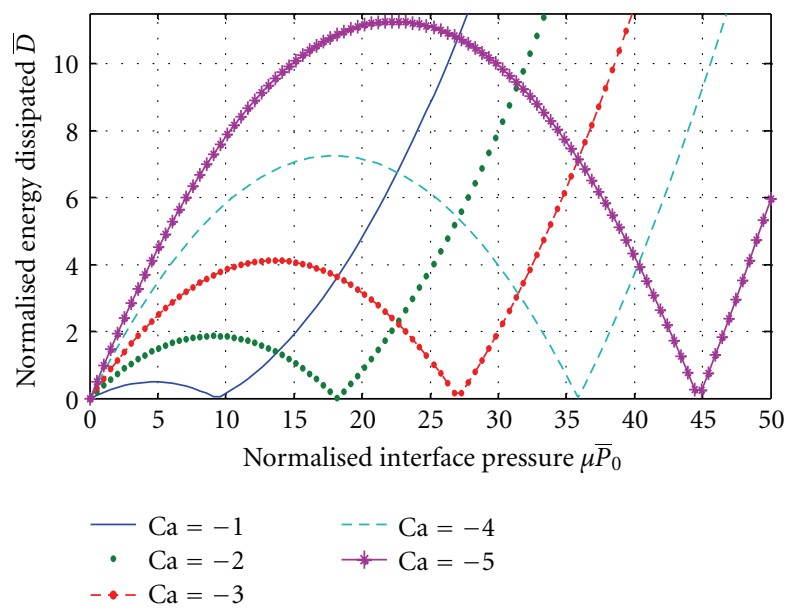

Figure 6: Energy dissipation profile for the case $\varepsilon=0$; $v_{1}=$ $0.35, v_{2}=0.25, \gamma_{\mathrm{eq}}=0.1, \Psi_{\mathrm{eq}}=0.001, \bar{\mu}_{1}=0.4, \bar{\mu}_{1}=0.2$, and frequency ratio $\eta=0.005$ and $\omega=10 \mathrm{KHz}$.

As stated above, we note that the value of $\kappa$ increases with decrement in energy dissipation for a prescribed value of the normalised pressure $\mu P$, part of the essence of this exercise is to know which is more efficacious between the ratio of laminate thicknesses $\Psi_{\text {eq }}$ and modulus of rigidity of laminate materials $\gamma_{\mathrm{eq}}$ for dissipation of vibration energy. The effect of the ratio of modulus of rigidity of laminate materials $\gamma_{\mathrm{eq}}$ is shown in Figures 6 and 7 for the same values of $\kappa$ and $\Psi_{\text {eq }}$, while the effect of laminate thicknesses $\Psi_{\text {eq }}$ is shown in Figures 9-11. Results indicate that more energy is dissipated as the value of either $\gamma_{\mathrm{eq}}$ or $\Psi_{\mathrm{eq}}$ increases. In particular, for values higher than 1, dissipation is higher than the results earlier reported in Olunloyo et al. [19].

It is clear in comparison that more energy can be dissipated by varying $\Psi_{\text {eq }}$ as against $\gamma_{\text {eq }}$ as seen in Figures 8 and 10 . By carefully selecting both $\Psi_{\text {eq }}$ and $\gamma_{\text {eq }}$ higher energy 


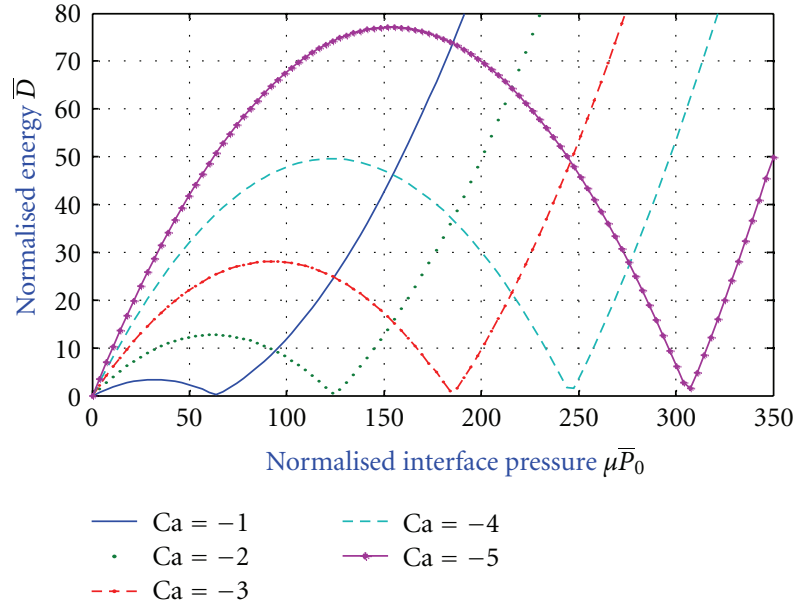

FIgURE 7: Energy dissipation profile for the case $\varepsilon=0$; $v_{1}=$ $0.35, v_{2}=0.25, \gamma_{\mathrm{eq}}=0.1, \Psi_{\mathrm{eq}}=0.001, \bar{\mu}_{1}=0.4, \bar{\mu}_{1}=0.2$, and frequency ratio $\eta=0.005$ and $\omega=100 \mathrm{KHz}$.

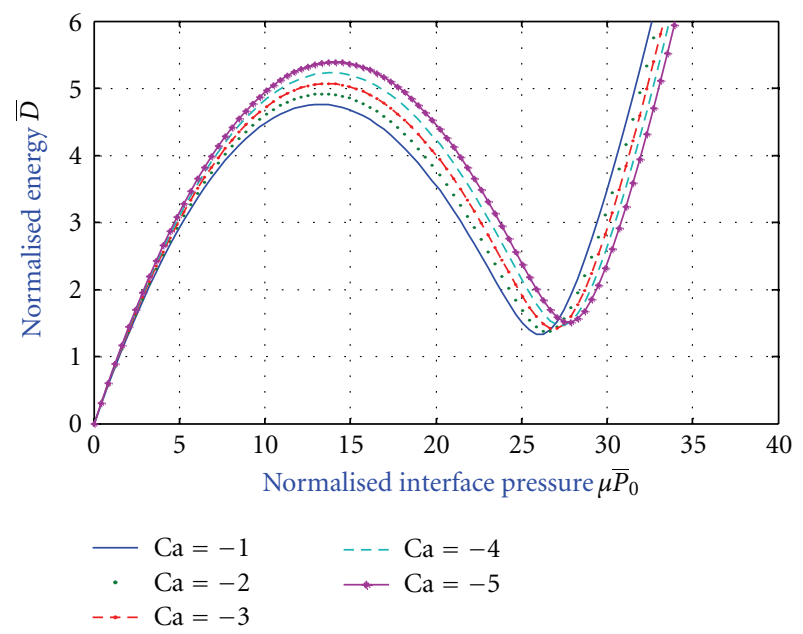

FIGURE 8: Energy dissipation profile for the case $\varepsilon=0 ; v_{1}=$ $0.35, v_{2}=0.25, \gamma_{\mathrm{eq}}=0.001, \Psi_{\mathrm{eq}}=0.1, \bar{\mu}_{1}=0.4, \bar{\mu}_{2}=0.2$, and frequency ratio $\eta=0.5$ and $\omega=8 \mathrm{KHz}$.

dissipation can be achieved than in any of the cases discussed above as seen from Figure 13, where $\Psi_{\mathrm{eq}}=\gamma_{\mathrm{eq}}=4 / 3$.

From engineering and economic points of view, it is much easier to have a hold on $\Psi$ than $\gamma$ since the former requires the preparation of laminates of certain thickness ratios. This is a straightforward matter, whereas it is sometimes a formidable problem to find appropriate laminate materials that will give a prescribed $\gamma_{\mathrm{eq}}$ ratio knowing that there might be other engineering, economic, or environmental requirements to be met in the use of such materials.

The pattern of results for energy dissipation also suggests that for effective energy dissipation there is a preferred arrangement of the laminates. The computation of $\Psi_{\text {eq }}$ shows that it is a composite laminate, which implies that the thickness of the upper laminate $h_{1}$ should always be less than that of the lower laminate $h_{2}$. Similarly, if the ratio of Young's

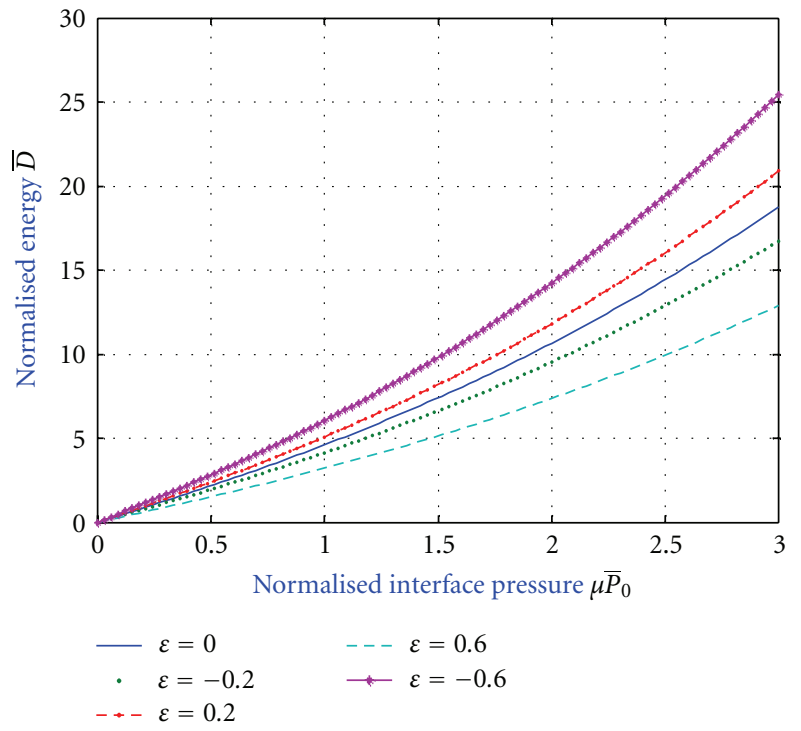

FIGURE 9: Energy dissipation profile for the case $\bar{C}_{a}=-1000 ; v_{1}=$ $0.35, v_{2}=0.25, \gamma_{\mathrm{eq}}=1, \Psi_{\mathrm{eq}}=1, \bar{\mu}_{1}=0.4, \bar{\mu}_{2}=0.2$, and frequency ratio $\eta=0.5$ and $\omega=8 \mathrm{KHz}$.

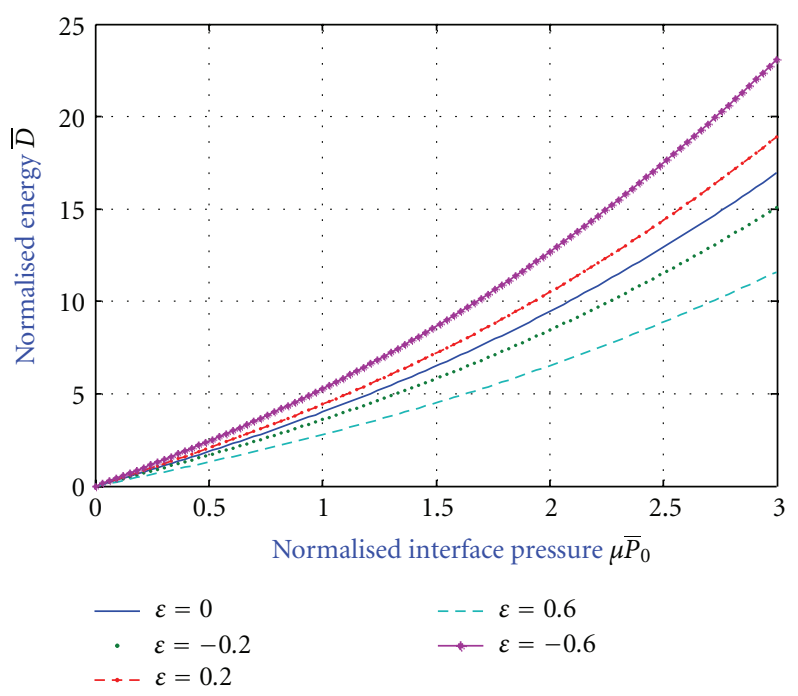

FIGURE 10: Energy dissipation profile for the case $\bar{C}_{a}=-1000 ; v_{1}=$ $0.25, v_{2}=0.5, \gamma_{\mathrm{eq}}=4 / 3, \Psi_{\mathrm{eq}}=1, \bar{\mu}_{1}=0.4, \bar{\mu}_{2}=0.2$, and frequency ratio $\eta=0.5$ and $\omega=8 \mathrm{KHz}$.

moduli of the laminates $\gamma_{\mathrm{eq}}=E_{\mathrm{eq} 2} / E_{\mathrm{eq} 1}$ has to be greater than 1 , the softer laminate is to be placed on top of the harder one. Thus when dealing with composite laminates, for optimal energy dissipation, it is imperative that the softer material (i.e., with Young's Modulus $E_{1}$ ) be placed on top of the harder laminate.

The general profile of logarithmic damping decrement curves for low frequency ratios clearly displays (cf. Figures 12 and 13) the pattern earlier reported by Masuko et al. 1973 [24]. This pattern is partly modified by the smart layers as observed from Figures 14 and 15. 


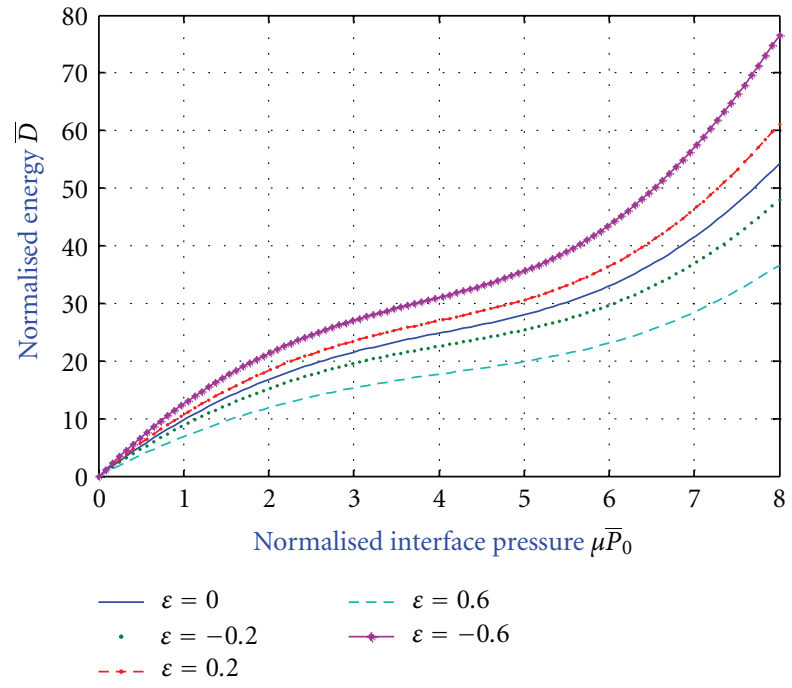

FIGURE 11: Energy dissipation profile for the case $\bar{C}_{a}=-1000 ; v_{1}=$ $0.25, v_{2}=0.5, \gamma_{\mathrm{eq}}=1, \Psi_{\mathrm{eq}}=4 / 3, \bar{\mu}_{1}=0.4, \bar{\mu}_{2}=0.2$, and frequency ratio $\eta=0.5$ and $\omega=8 \mathrm{KHz}$.

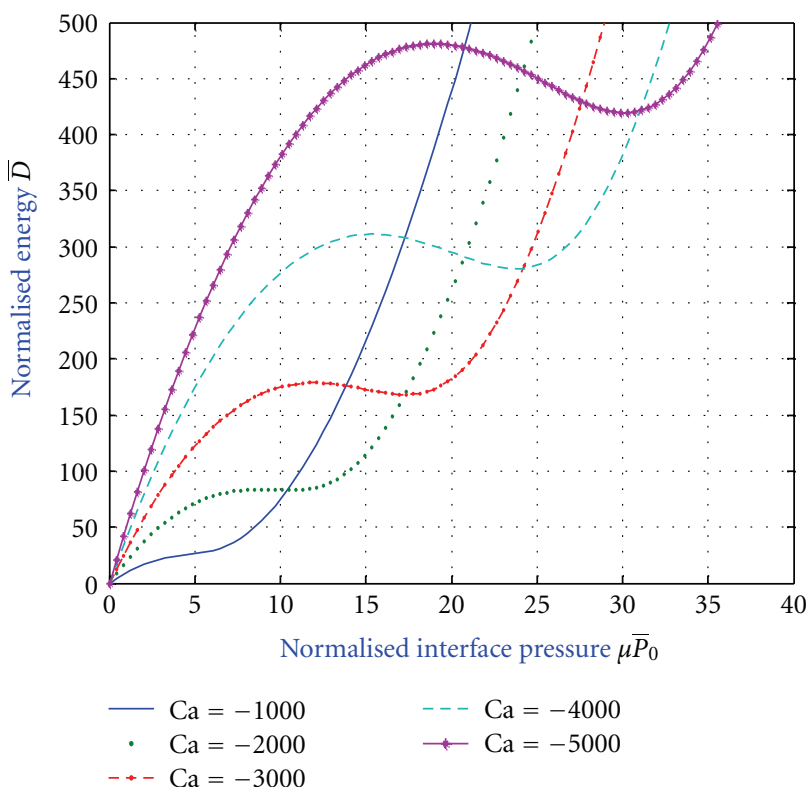

FIGURE 12: Energy dissipation profile for the case $\varepsilon=0 ; v_{1}=$ $0.35, v_{2}=0.25, \gamma_{\mathrm{eq}}=4 / 3, \Psi_{\mathrm{eq}}=4 / 3, \bar{\mu}_{1}=0.4, \bar{\mu}_{2}=0.2$, and frequency ratio $\eta=0.5$ and $\omega=8 \mathrm{KHz}$.

The modulating effects of playing with the values of $\gamma_{\mathrm{eq}}$ and $\Psi_{\text {eq }}$ for moderate values of $\eta$ is also noted in Figures 16 and 17. Furthermore, as the value of the frequency ratio $\eta$ gets large and beyond resonance as in Figures 16 and 17, the nature of the logarithmic damping decrement profile transits from the results of Masuko et al. [24] to those of Nanda and Behera [15]. Irrespective of the value of $\varepsilon$, the logarithmic damping decrement $\delta$ decreases as the value of $\mu P$ increases reflecting a higher normal force at the interface of the laminates. Thus, the effect of the smart layers is to moderate

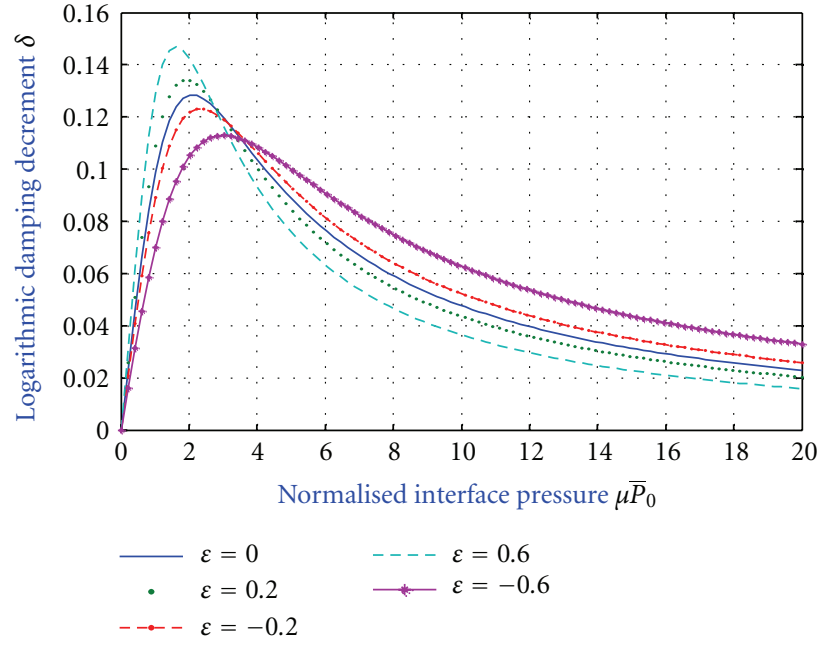

FIgURE 13: Logarithmic damping decrement profile for the case $\bar{C}_{a}=-1000 ; v_{1}=0.35, v_{2}=0.25, \gamma_{\mathrm{eq}}=100, \Psi_{\text {eq }}=0.1, \bar{\mu}_{1}=$ $0.4, \bar{\mu}_{2}=0.1$, and frequency ratio $\eta=0.05$ and $\omega=8 \mathrm{KHz}$.

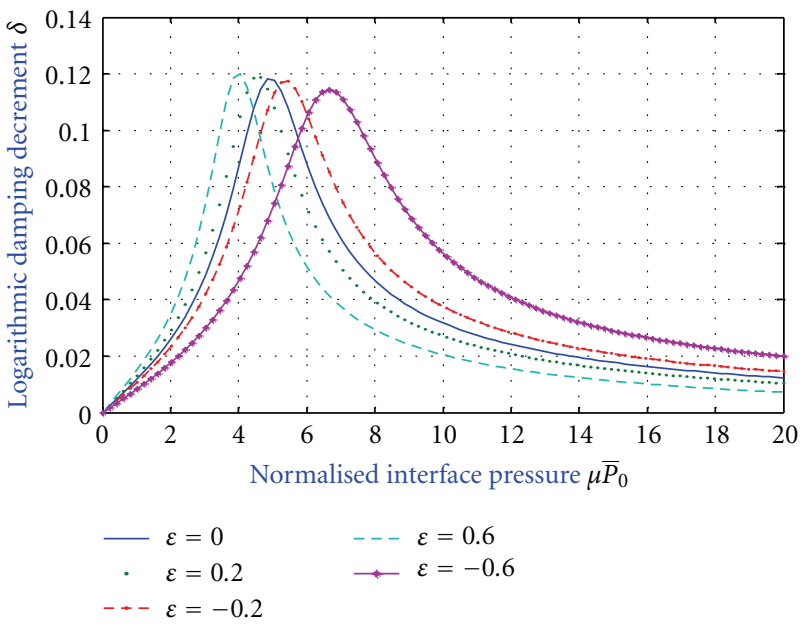

Figure 14: Logarithmic damping decrement profile for the case $\bar{C}_{a}=-1000 ; v_{1}=0.35, v_{2}=0.25, \gamma_{\mathrm{eq}}=100, \Psi_{\mathrm{eq}}=0.1, \bar{\mu}_{1}=$ $0.4, \bar{\mu}_{2}=0.2$, and frequency ratio $\eta=0.85$ and $\omega=8 \mathrm{KHz}$.

the logarithmic damping profile. The significance of these results depends upon the practical relevance of the regime of frequency ratio of the situation under consideration.

\section{Summary and Conclusion}

In this paper, vibration and noise dissipation in ships FPS and FPSO structures using layered smart systems is discussed. In the formulated boundary value problem, the physics derives from energy dissipation through press fit joints or interface in an environment of linear pressure distribution. By employing the integral transform approach,

(a) explicit solutions for the response, slip, optimal pressure, and dissipated energy (i.e., $W, \Delta u, P_{\mathrm{opt}}$ 


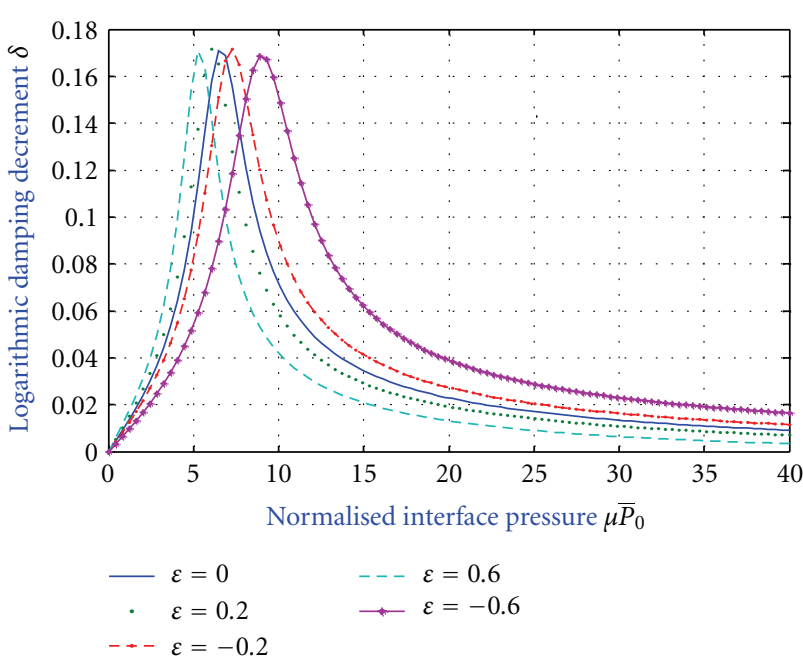

FIGURE 15: Logarithmic damping decrement profile for the case $\bar{C}_{a}=-5 ; v_{1}=0.25, v_{2}=0.5, \gamma_{\mathrm{eq}}=500, \Psi_{\mathrm{eq}}=0.1$, and frequency ratio $\eta=0.85$ and $\omega=8 \mathrm{KHz}$.

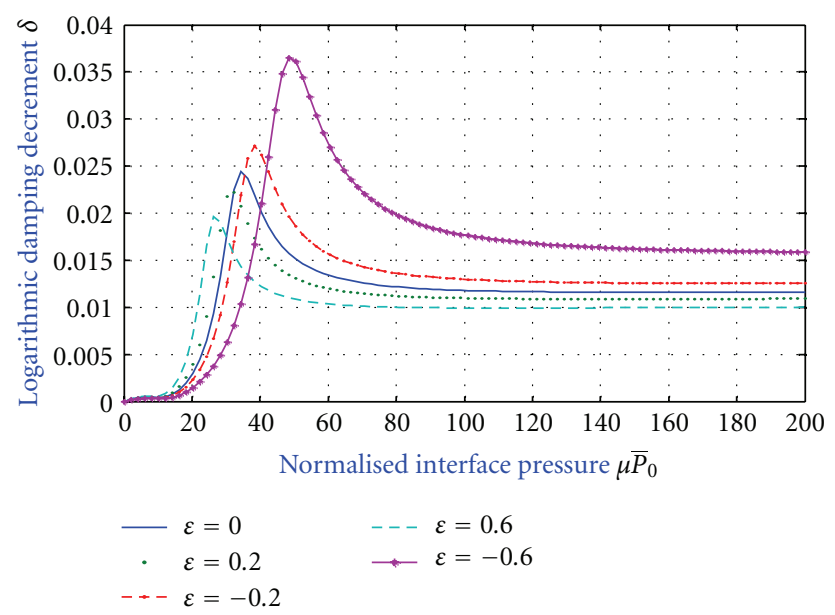

FIGURE 16: Logarithmic damping decrement profile for the case $\bar{C}_{a}=-1000 ; v_{1}=0.25, v_{2}=0.5, \gamma_{\mathrm{eq}}=0.5, \Psi_{\mathrm{eq}}=1$, and frequency ratio $\eta=2.5$ and $\omega=8 \mathrm{KHz}$.

and $\bar{D}$, resp.) under periodic forcing functions were computed,

(b) the amount of slip damping as influenced by the linear pressure distribution across the laminate interface, the relative thicknesses of the laminates members, and their elasticity moduli was evaluated,

(c) the ordering of the laminates is equally significant, as the pattern of results for energy dissipation indicates that there is a preferred sandwich geometry,

(d) in particular, for optimal energy dissipation the harder material (i.e., with Young's Modulus $E_{2}$ ) is placed under the softer laminate,

(e) similarly, more energy is dissipated when the harder laminate is thicker than the softer one,

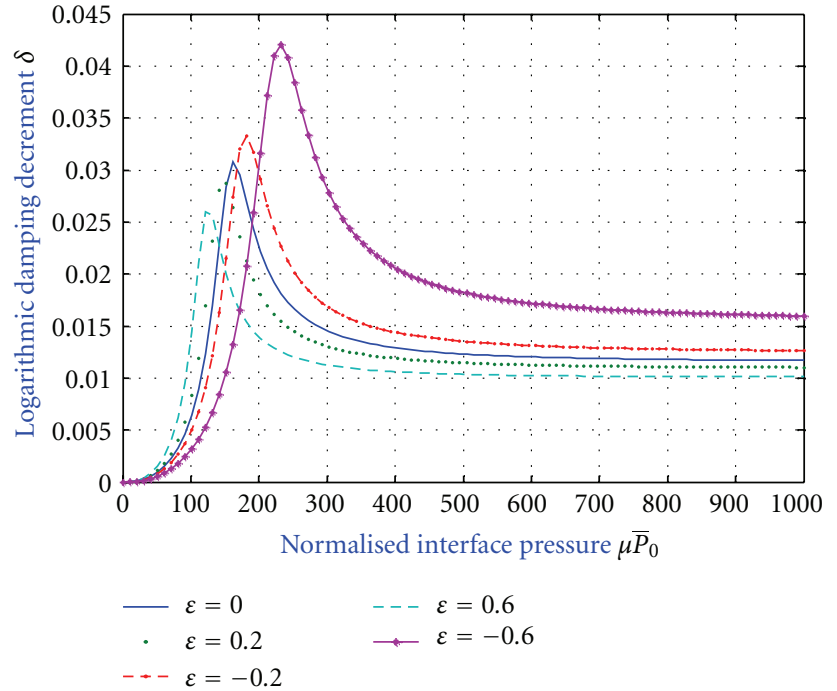

FIGURE 17: Logarithmic damping decrement profile for the case $\bar{C}_{a}=-1000 ; v_{1}=0.35, v_{2}=0.25, \gamma_{\mathrm{eq}}=0.5, \Psi_{\mathrm{eq}}=1$, and frequency ratio $\eta=3.5$ and $\omega=8 \mathrm{KHz}$.

(f) with respect to the logarithmic damping decrement, results show that for small values of the frequency ratio $\eta$ there is a close agreement between these results and those reported earlier in Masuko et al. [24], while, for high values of $\eta$, the nature of profiles highlighted results in Olunloyo et al. [19], thus, behavioural pattern predicted by earlier workers can be recovered in appropriate limits of the present work,

(g) the results also show that by incorporating smart structures, the level of damping can be significantly increased.

\section{Nomenclature}

$d / d x$ : Differential operator

$x$ : Space coordinate along the beam-plate interface

$z$ : Space coordinate perpendicular to the beam-plate interface

E: $\quad$ Modulus of rigidity

I: $\quad$ Moment of inertia

$W: \quad$ Transverse dynamic response

$b$ : Width of laminated beam plate

$h$ : Depth of laminated beam plate

$t$ : Time coordinate

$P$ : Clamping pressure at the interface of the laminates

$F(t)$ : Applied end force

$L: \quad$ Length of laminated beam plate

$u_{1}$ : Longitudinal displacement of the upper laminate at interface 
$u_{2}$ : Longitudinal displacement of the lower laminate at interface

$W^{F}$ : Transverse response in Fourier plane.

\section{Greek Letters}

$\mu$ : Coefficient of friction at the interface of laminated viscoelastic laminates

$\bar{\mu}: \quad$ Hysteretic damping constant

$\gamma$ : $\quad$ Structural damping coefficient of viscoelastic laminates

$\rho: \quad$ Density of laminate material

$\tau_{x z}$ : Shear stress at the interface of the laminates

$\left(\sigma_{x}\right)_{1}$ : Bending stress at the upper half of the laminates

$\left(\sigma_{x}\right)_{2}$ : Bending stress at the lower half of the laminates

$\xi: \quad$ Dummy variable

$v$ : Poisson's ratio.

\section{References}

[1] T. Moan, E. Ayala-Uraga, and X. Wang, "Reliability-based service life assessment of FPSO structures," Proceedings of the Society of Naval Architects and Marine Engineers, vol. 112, pp. 314-342, 2004.

[2] B. J. Lazan, Damping of Materials and Members in Structural Mechanics, Pergamon Press, Oxford, UK, 1968.

[3] B. C. Nakra, "Vibration control in machines and structures using viscoelastic damping," Journal of Sound and Vibration, vol. 211, no. 3, pp. 449-465, 1998.

[4] C. T. Sun and Y. P. Lu, Vibration Damping of Structural Elements, Prentice Hall, Upper Saddle River, NJ, USA, 1995.

[5] L. Garibaldi and H. N. Onah, Viscoelastic Material Damping Technology, Beechis Osiride, Torino, Italy, 1996.

[6] D. J. Meador and D. J. Mead, Passive Vibration Control, John Wiley \& Sons, New York, NY, USA, 1999.

[7] E. J. Vydra and J. P. Shogren, "Noise and noise reducing materials," Society of Automotive Engineers Paper 931267, 1993.

[8] M. D. Rao, "Recent applications of viscoelastic damping for noise control in automobiles and commercial airplanes," Journal of Sound and Vibration, vol. 262, no. 3, pp. 457-474, 2003.

[9] R. O. S. Moheimani, R. H. Pota, and R. I. Petersen, "Spatial control for active vibration control of piezoelectric laminates," in Proceedings of the 37th IEEE Conference on Decision and Control (CDC'98), pp. 4308-4313, December 1998.

[10] T. Bailey and J. E. Hubbard, "Distributed piezoelectric-polymer active vibration control of a cantilever beam," Journal of Guidance, Control, and Dynamics, vol. 8, no. 5, pp. 605-611, 1985.

[11] T. E. Alberts, T. V. DuBois, and H. R. Pota, "Experimental verification of transfer functions for a slewing piezoelectric laminate beam," Control Engineering Practice, vol. 3, no. 2, pp. 163-170, 1995.

[12] H. S. Tzou, "Integrated distributed sensing and active vibration suppression of flexible manipulators using distributed piezoelectrics," Journal of Robotic Systems, vol. 6, no. 6, pp. 745-767, 1989.
[13] E. Goodman and J. H. Klumpp, "Analysis of slip damping with reference to turbine blade vibration," Journal of Applied Mechanics, vol. 23, pp. 421-429, 1956.

[14] B. K. Nanda, Study of damping in structural members under controlled dynamic slip, Ph.D. thesis, 1992.

[15] B. K. Nanda and A. K. Behera, "Study on damping in layered and jointed structures with uniform pressure distribution at the interfaces," Journal of Sound and Vibration, vol. 226, no. 4, pp. 607-624, 1999.

[16] O. Damisa, V. O. S. Olunloyo, C. A. Osheku, and A. A. Oyediran, "The static analysis of slip damping with clamped laminated beams," European Journal of Scientific Research, vol. 17, no. 4, pp. 455-476, 2007.

[17] V. O. S. Olunloyo, O. Damisa, C. A. Osheku, and A. A. Oyediran, "Further results concerning the static analysis of slip damping with clamped laminated beams," European Journal of Scientific Research, vol. 17, no. 4, pp. 491-508, 2007.

[18] O. Damisa, V. O. S. Olunloyo, C. A. Osheku, and A. A. Oyediran, "Dynamic analysis of slip damping in clamped layered beams with non-uniform pressure distribution at the interface," Journal of Sound and Vibration, vol. 309, no. 3-5, pp. 349-374, 2008.

[19] V. O. S. Olunloyo, C. A. Osheku, and O. Damisa, "Vibration damping in structures with layered viscoelastic beam-plate," ASME Journal of Vibration and Acoustics, vol. 130, no. 6, Article ID 061002, pp. 1-26, 2008.

[20] V. O. S. Olunloyo, C. A. Osheku, and F. Agboola, "Vibration damping and active noise control in ships and floating structures," in Proceedings of the 26th ASME International Conference on Offshore Mechanics and Arctic Engineering (OMAE '07), vol. 1, pp. 3-17, June 2007.

[21] V. O. S. Olunloyo, C. A. Osheku, and A. A. Oyediran, "Dynamic response interaction of vibrating offshore pipeline on moving seabed," ASME Journal of Offshore Mechanics and Arctic Engineering, vol. 129, no. 2, pp. 107-120, 2007.

[22] V. O. S. Olunloyo, A. A. Oyediran, and C. A. Osheku, "Dynamic response interaction of vibrating offshore pipeline and moving seabed with varying geological and geo-mechanical properties," ASME Journal of Offshore Mechanics and Arctic Engineering, vol. 3, pp. 163-184, 2004.

[23] V. O. S. Olunloyo, C. A. Osheku, and O. Damisa, "On the non-linear analysis of pipeline-soil interaction dynamics on the ocean bed," in Proceedings of the 25th ASME International Conference on Offshore Mechanics and Arctic Engineering (OMAE '06), vol. 4, pp. 183-196, June 2006.

[24] M. Masuko, Y. Ito, and K. Yoshida, "Theoretical analysis for a damping ratio of a jointed cantibeam," Bulletin of the Japan Society of Mechanical Engineers, vol. 16, no. 99, pp. 1421-1432, 1973.

[25] C. K. Lee, Piezoelectric laminates for torsional and bending modal control: theory and experiment, Ph.D. thesis, Cornell University, 1987.

[26] H. H. Cudney, Distributed structural control using multilayered piezoelectric actuators, Ph.D. thesis, Suny Buffalo, New York, NY, USA, 1989.

[27] J. Pan, C. H. Hansen, and S. D. Snlyer, "A study of the response of a simply supported beam to excitation by a piezoelectric actuator," in Proceedings of the Conference on Recent Advances in Active Control of Sound and Vibration, p. 349, Virginia Polytechnic Institute and State University, Virginia, Va, USA, 1991.

[28] C. K. Lee and F. C. Moon, "Modal sensors/actuators," Transactions of the ASME, and Journal of Applied Mechanics, vol. 57, pp. 434-441, 1991. 
[29] A. Baz and S. Poh, "Performance of an active control system with piezoelectric actuators," Journal of Sound and Vibration, vol. 126, no. 2, pp. 327-343, 1988.

[30] H. R. Pota and T. E. Alberts, "Multivariable transfer functions for a slewing piezoelectric laminate beam," Transactions of the ASME Journal of Dynamic Systems, Measurement and Control, vol. 117, no. 3, pp. 352-359, 1994. 

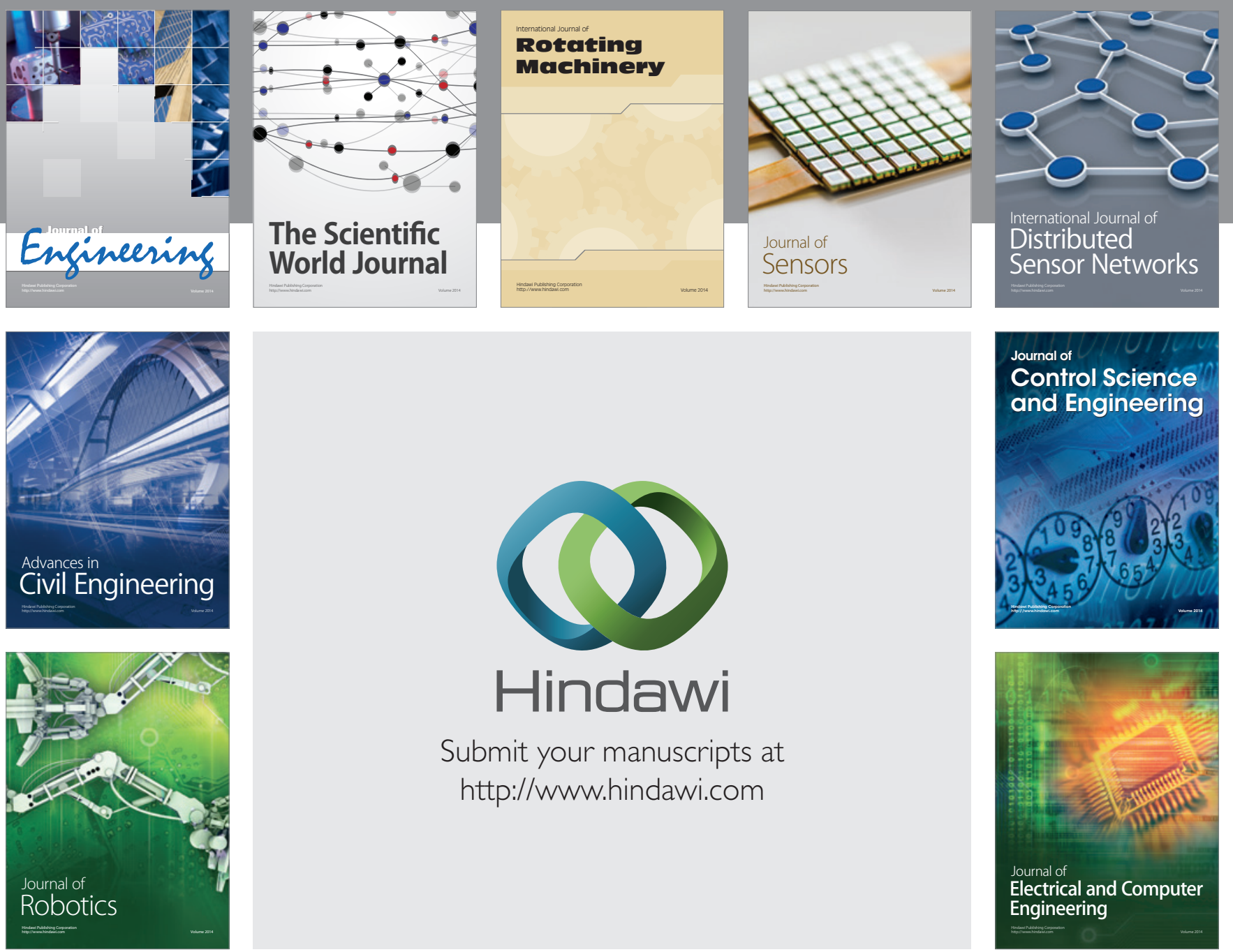

Submit your manuscripts at

http://www.hindawi.com
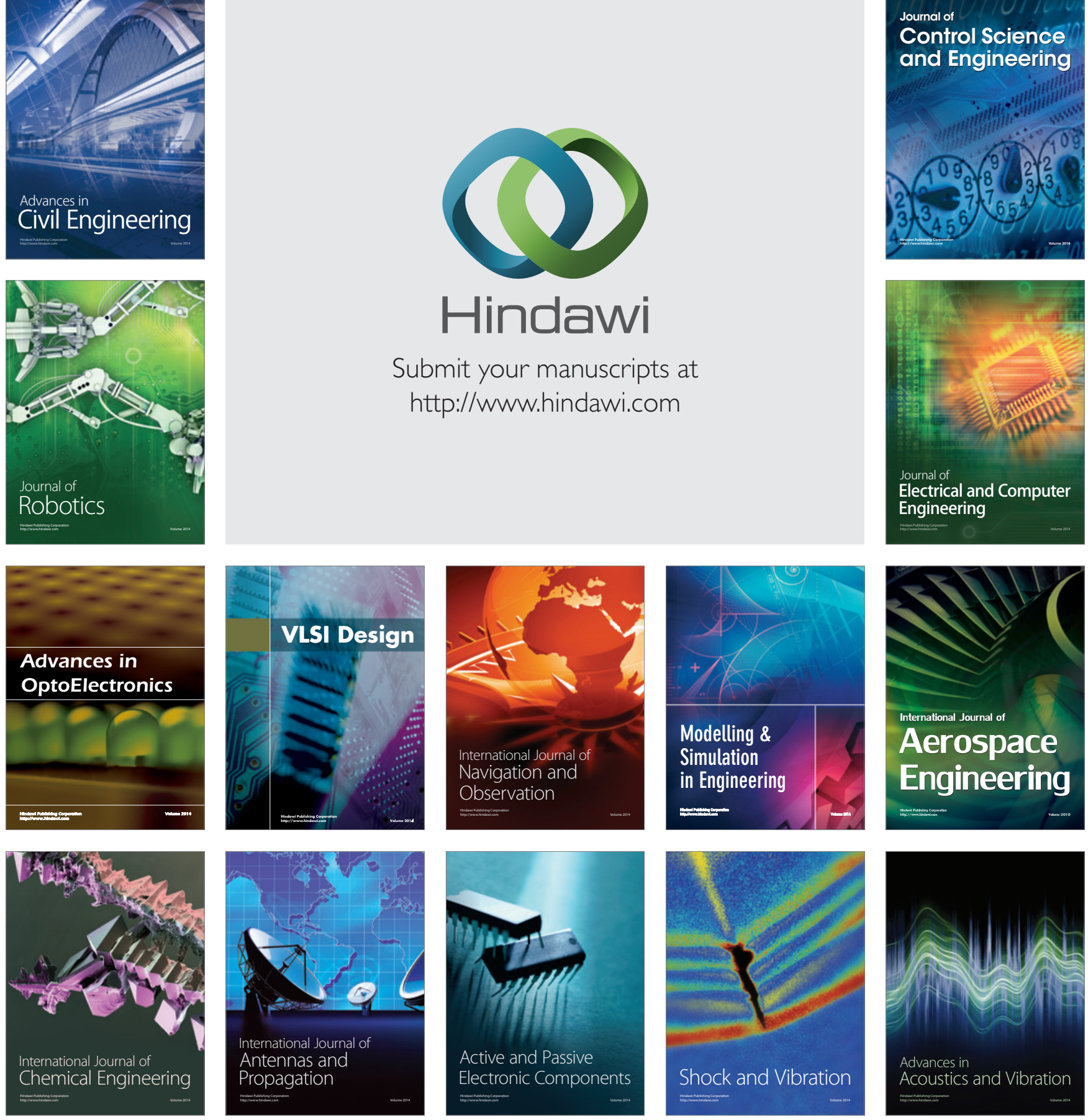